\title{
New long-lived particle searches in heavy-ion collisions at the LHC
}

\author{
Marco Drewes๑, ${ }^{*}$ Andrea Giammanco $\odot,{ }^{\dagger}$ Jan Hajer $\odot,{ }^{*}$ and Michele Lucente ${ }^{\S}$ \\ Centre for Cosmology, Particle Physics and Phenomenology, Université catholique de Louvain, \\ Louvain-la-Neuve B-1348, Belgium
}

(Received 9 August 2019; accepted 7 February 2020; published 3 March 2020)

\begin{abstract}
We show that heavy-ion collisions at the LHC provide a promising environment to search for signatures with displaced vertices in well-motivated new physics scenarios. Compared to proton collisions, they offer several advantages: (i) the number of parton level interactions per collision is larger, (ii) there is no pileup, (iii) the lower instantaneous luminosity compared to proton collisions allows one to operate the LHC experiments with very loose triggers, and (iv) there are new production mechanisms that are absent in proton collisions We focus on the third point and show that the modification of the triggers alone can increase the number of observable events by orders of magnitude if the long-lived particles are predominantly produced with low transverse momentum. Our results show that collisions of ions lighter than lead are well motivated from the viewpoint of searches for new physics. We illustrate this for the example of heavy neutrinos in the Neutrino Minimal Standard Model.
\end{abstract}

DOI: 10.1103/PhysRevD.101.055002

\section{INTRODUCTION}

The search for new elementary particles is one of the most pressing quests in contemporary physics. While the Standard Model (SM) of particle physics describes phenomena ranging from the inner structure of the proton to the spectra of distant galaxies with an astonishing accuracy, there is unambiguous proof that it does not constitute a complete description of nature, including the dark matter puzzle, the excess of matter over antimatter in the observable Universe, and flavor-changing oscillations among the known neutrino states. In addition to these facts, there are a number of observations that in principle can be explained by the SM and the theory of general relativity, but only by choosing values of the fundamental parameters that are considered unsatisfactory by many theorists, including the electroweak hierarchy problem [1], the flavor puzzle [2], strong CP problem [3-5], and the value of the vacuum energy or cosmological constant [6,7]. Before the LHC was turned on it was widely believed, based on theoretical arguments [8], that the new particles that are responsible for these phenomena have masses only slightly above the electroweak scale and interact with the SM particles at a

\footnotetext{
*marco.drewes@uclouvain.be †andrea.giammanco@uclouvain.be \#jan.hajer@uclouvain.be

\$michele.lucente@uclouvain.be
}

Published by the American Physical Society under the terms of the Creative Commons Attribution 4.0 International license. Further distribution of this work must maintain attribution to the author(s) and the published article's title, journal citation, and DOI. Funded by SCOAP. rate that allows one to produce them copiously at the LHC. However, the nonobservation of any new elementary particles beyond the Higgs boson predicted by the SM has broadened the scope of experimental searches at CERN [9] and other laboratories around the world. In the present situation it seems mandatory, from an experimental viewpoint, to minimize the dependence on specific theory frameworks and "turn every stone" in the search for new physics. It has recently been pointed out that this strategy should also include the use of data from heavy-ion collisions to search for new phenomena [10].

In the present work we study the possibility to search for long-lived particles (LLPs) in heavy-ion collisions. LLPs appear in a wide range of models of physics beyond the SM [11]. They can owe their longevity to a combination of different mechanisms that are known to be at work in the $\mathrm{SM}$, including symmetries, the mass spectrum, and small coupling constants. In collider experiments they reveal themselves with displaced signatures in the detectors. The displacement makes it possible to distinguish the signal from the many track environment that is created in a heavyion collision, because all primary tracks from primary SM interactions originate from within the microscopic volume of the two colliding nuclei. Our proposal is driven by the approach to fully exploit the discovery potential of the existing detectors to make optimal use of CERN's resources and infrastructure. This approach is complementary to proposals that aim to improve the sensitivity of the LHC to LLPs by adding new detectors, including the recently approved FASER experiment [12] and other dedicated detectors, such as MATHUSLA [13-15], CODEX-b [16], and Al3X [17]. A summary of our main results is presented in Ref. [18]. 
TABLE I. Cross sections for different heavy ions based on [24]. Here $M_{\text {ion }}$ indicates the mass of the ion and $\sqrt{s_{X X}}$ the nucleon-nucleon center of mass energy achievable at the LHC. The total cross section is the sum of the electromagnetic dissociation (EMD), the boundfree pair production (BFPP), and the hadronic cross section $\sigma_{\mathrm{tot}}=\sigma_{\mathrm{EMD}}+\sigma_{\mathrm{BFPP}}+\sigma_{\text {had }} . \sigma_{W}^{\mathrm{XX}}$ indicates the $W$-boson cross section per nucleon. For this illustrative purpose of this table we estimate $\sigma_{W}^{\mathrm{XX}}$ from $p p \rightarrow W^{ \pm} \rightarrow \mu^{ \pm} \nu$ with $p_{T}(\mu)>5 \mathrm{GeV}$, calculated at next-toleading order (NLO) using MadGraph5_aMC@NLO [28], while we properly simulate heavy-ion collisions as described below to obtain the results shown in Fig. 5. $\sigma_{W}$ indicates the nuclear cross section, estimated by scaling $\sigma_{W}^{\mathrm{XX}}$ with $A^{2}$.

\begin{tabular}{|c|c|c|c|c|c|c|c|c|}
\hline & $M_{\text {ion }}(\mathrm{GeV})$ & $\sqrt{s_{X X}}(\mathrm{TeV})$ & $\sigma_{\mathrm{EMD}}(\mathrm{b})$ & $\sigma_{\mathrm{BFPP}}(\mathrm{b})$ & $\sigma_{\text {had }}(\mathrm{b})$ & $\sigma_{\text {tot }}(\mathrm{b})$ & $\sigma_{W}^{\mathrm{XX}}(\mathrm{nb})$ & $\sigma_{W}(\mu \mathrm{b})$ \\
\hline${ }_{1}^{1} \mathrm{H}$ & 0.931 & 14.0 & 0 & 0 & 0.071 & 0.071 & 20.3 & 0.0203 \\
\hline${ }_{8}^{16} \mathrm{O}$ & 14.9 & 7.00 & 0.074 & $24 \times 10^{-6}$ & 1.41 & 1.47 & 10.0 & 2.56 \\
\hline${ }_{18}^{40} \mathrm{Ar}$ & 37.3 & 6.30 & 1.2 & 0.0069 & 2.6 & 3.81 & 8.92 & 14.3 \\
\hline${ }_{20}^{40} \mathrm{Ca}$ & 37.3 & 7.00 & 1.6 & 0.014 & 2.6 & 4.21 & 10.0 & 16.0 \\
\hline $\begin{array}{l}78 \\
{ }_{36}^{7} \\
\mathrm{Kr}\end{array}$ & 72.7 & 6.46 & 12 & 0.88 & 4.06 & 17.0 & 9.16 & 55.7 \\
\hline${ }_{36}^{84} \mathrm{Kr}$ & 78.2 & 6.00 & 13 & 0.88 & 4.26 & 18.2 & 8.43 & 59.5 \\
\hline${ }_{54}^{129} \mathrm{Xe}$ & 120 & 5.86 & 52 & 15 & 5.67 & 72.7 & 8.22 & 137 \\
\hline $\begin{array}{l}208 \mathrm{~Pb} \\
\end{array}$ & 194 & 5.52 & 220 & 280 & 7.8 & 508 & 7.69 & 333 \\
\hline
\end{tabular}

\section{HEAVY-ION COLLISIONS AT THE LHC}

For an equal number of collisions and equal center-ofmass energy per nucleon, collisions of heavier nuclei guarantee larger hard-scattering cross sections than proton $(p p)$ collisions, thanks to the enhancement factor of $\approx A^{2}$ in the number of parton level interactions, where $A$ is the mass number of the isotope under consideration. In the case of lead isotopes $\left({ }_{82}^{208} \mathrm{~Pb}\right)$ accelerated in the LHC, $A=208$ provides an enhancement of 4 orders of magnitude. However, there are several drawbacks.

(1) The collision energy per nucleon in heavy-ion collisions is smaller than in proton collisions. The design LHC beam energy is limited to $2.76 \mathrm{TeV}$ for $\mathrm{Pb}$ ions (corresponding to a center-of-mass energy per nucleon of $\sqrt{s_{\mathrm{XX}}}=5.52 \mathrm{TeV}$ in $\mathrm{PbPb}$ collisions), to be compared with $7 \mathrm{TeV}$ for proton beams (hence $\sqrt{s_{p p}}=14 \mathrm{TeV}$ ). These design values are expected to be reached during Run 3. The scaling factor $\left.\sigma^{p p}\right|_{14 \mathrm{TeV}} /\left.\sigma^{\mathrm{XX}}\right|_{5.52 \mathrm{TeV}}$ grows as a function of the particle masses in the final state of the hard process under consideration [19], and it is typically larger for gluon-initiated processes than for quarkantiquark collisions. For instance, for top quark pair $(t \bar{t})$ production the drop between 14 and $5.52 \mathrm{TeV}$ is of 1 order of magnitude [20] due to the large mass of the top quark. However, the $W$-boson production cross section per nucleon is only reduced by a factor of around 2.5 (see Table I). The reduction factor is around 2.3 for $B$ mesons [21-23], which are lighter but whose production at these LHC energies is mostly initiated by gluon-gluon fusion (opposed to $W$ bosons, which are mostly created by quarkantiquark annihilation).

(2) Heavy-ion collisions are characterized by the production of a very large particle multiplicity, and in particular a very large multiplicity of charged-particle tracks, which poses challenges for data acquisition and data analysis to the multipurpose LHC experiments. While a large track multiplicity generates a strong background for prompt signatures, the decay of feebly interacting LLPs produces displaced tracks at macroscopic distances from the interaction point that can be easily distinguished from the tracks that originate from the primary interaction at the collision point. We address this point in Sec. III.

(3) The instantaneous luminosity in heavy-ion runs is limited to considerably lower values compared to $p p$ collisions, see Table II. The LHC delivered $1.8 \mathrm{nb}^{-1}$ of collisions to the ATLAS and CMS detectors during the latest $\mathrm{PbPb}$ run in late 2018, and $10 \mathrm{nb}^{-1}$ are expected to be accumulated during the high-luminosity phase of the accelerator (HLLHC) [24]. In terms of sheer numbers, this cannot compete with the size of the $p p$ data samples even if the $A^{2}$ enhancement due to nucleon-nucleon combinatorics is taken into account. This poses the strongest limitation when searching for rare phenomena in heavy-ion data. We discuss these luminosity limitations in some detail in Sec. IV.

(4) Heavy-ion runs at the LHC are comparably short. In the past not more than one month has been allocated in the yearly schedule, as opposed to around six months in the $p p$ case. This is not a fundamental restriction, and one can imagine that the sharing of time may change in the future depending on the priorities of the LHC experiments. In the following we compare the sensitivity per equal running time, given a realistic instantaneous luminosity, in order to remain independent of possible changes in the planning.

On the other hand, there are key advantages in heavy-ion collisions.

(i) The aforementioned number of parton level interactions per collision is larger. 
TABLE II. Luminosities for collisions of different heavy ions based on [24] for three choices of the scaling parameter $p$ [see definition (9)]. $\mathcal{L}_{0}$ is the peak luminosity, $\tau_{b}$ the optimal beam lifetime, and $\mathcal{L}_{\text {ave }}$ the optimized average luminosity. The last column contains the ratio between the number of events $N_{\mathrm{XX}}=L \sigma_{W}$ in XX and $p p$ production, where $L$ is the integrated luminosity [see definition (5)] and $\sigma_{W}$ is given in I. Following [24], we use an optimistic turnaround time of $1.25 \mathrm{~h}$, which we compensate for in the case of heavy-ion collisions by assuming that the useful run-time is only half of the complete run-time.

\begin{tabular}{|c|c|c|c|c|c|c|c|c|c|c|c|c|}
\hline & \multicolumn{4}{|c|}{ Pessimistic $(p=1)$} & \multicolumn{4}{|c|}{ Realistic $(p=1.5)$} & \multicolumn{4}{|c|}{ Optimistic $(p=1.9)$} \\
\hline & $\begin{array}{c}\mathcal{L}_{0} \\
\left(\mu \mathrm{bs}^{-1}\right)\end{array}$ & $\begin{array}{l}\tau_{b} \\
(\mathrm{~h})\end{array}$ & $\begin{array}{c}\mathcal{L}_{\text {ave }} \\
\left(\mu \mathrm{bs}^{-1}\right)\end{array}$ & $\begin{array}{c}N_{\mathrm{XX}} / N_{p p} \\
{[1]}\end{array}$ & $\begin{array}{c}\mathcal{L}_{0} \\
\left(\mu \mathrm{bs}^{-1}\right)\end{array}$ & $\begin{array}{l}\tau_{b} \\
(\mathrm{~h})\end{array}$ & $\begin{array}{c}\mathcal{L}_{\text {ave }} \\
\left(\mu \mathrm{bs}^{-1}\right)\end{array}$ & $\begin{array}{c}N_{\mathrm{XX}} / N_{p p} \\
{[1]}\end{array}$ & $\begin{array}{c}\mathcal{L}_{0} \\
\left(\mu \mathrm{bs}^{-1}\right)\end{array}$ & $\begin{array}{l}\tau_{b} \\
(\mathrm{~h})\end{array}$ & $\begin{array}{c}\mathcal{L}_{\text {ave }} \\
\left(\mu \mathrm{bs}^{-1}\right)\end{array}$ & $\begin{array}{c}N_{\mathrm{XX}} / N_{p p} \\
{[1]}\end{array}$ \\
\hline${ }_{1}^{1} \mathrm{H}$ & $21.0 \times 10^{3}$ & 75.0 & $15.0 \times 10^{3}$ & 1 & $21.0 \times 10^{3}$ & 75.0 & $15.0 \times 10^{3}$ & 1 & $21.0 \times 10^{3}$ & 75.0 & $15.0 \times 10^{3}$ & 1 \\
\hline${ }_{8}^{16} \mathrm{O}$ & 1.43 & 52.6 & 1.07 & 0.0082 & 14.6 & 16.4 & 8.97 & 0.0688 & 94.3 & 6.48 & 45.5 & 0.349 \\
\hline${ }_{10}^{\circ} \mathrm{Ar}$ & 0.282 & 45.8 & 0.208 & 0.00889 & 1.29 & 21.5 & 0.837 & 0.0358 & 4.33 & 11.7 & 2.46 & 0.105 \\
\hline${ }_{20}^{40} \mathrm{Ca}$ & 0.229 & 46.0 & 0.168 & 0.00811 & 0.937 & 22.7 & 0.615 & 0.0296 & 2.90 & 12.9 & 1.69 & 0.0811 \\
\hline${ }_{36}^{78} \mathrm{Kr}$ & 0.0706 & 20.6 & 0.0454 & 0.00758 & 0.161 & 13.6 & 0.0948 & 0.0158 & 0.311 & 9.80 & 0.169 & 0.0282 \\
\hline${ }_{36}^{84} \mathrm{Kr}$ & 0.0706 & 19.2 & 0.0448 & 0.00797 & 0.161 & 12.7 & 0.0933 & 0.0166 & 0.311 & 9.15 & 0.166 & 0.0296 \\
\hline${ }_{54}^{129} \mathrm{Xe}$ & 0.0314 & 7.20 & 0.156 & 0.00637 & 0.0476 & 5.84 & 0.0222 & 0.00908 & 0.0665 & 4.94 & 0.0294 & 0.0120 \\
\hline $\begin{array}{l}208 \mathrm{~Pb} \\
82\end{array}$ & 0.0136 & 1.57 & $3.79 \times 10^{-3}$ & 0.00379 & 0.0136 & 1.57 & $3.8 \times 10^{-3}$ & 0.00379 & 0.0136 & 1.57 & $3.8 \times 10^{-3}$ & 0.00379 \\
\hline
\end{tabular}

(ii) There is no pileup in heavy-ion collisions. In collisions of high intensity proton beams pileup leads to tracks that originate from different points in the same bunch crossing and creates a considerable background for displaced signatures. In heavy-ion collisions the probability of misidentifying the primary vertex is negligible. Hence, heavy-ion collisions can provide a cleaner environment to search for signatures stemming from the decay of LLPs when pileup is a problem, see Fig. 1.

(iii) The lower instantaneous luminosity makes it possible to operate ATLAS and CMS with significantly lower trigger thresholds. This, e.g., allows one to detect events with comparably low transverse momentum $p_{T}$ in scenarios involving light mediators or when the LLPs are produced in the decay of mesons.

(iv) In heavy-ion collisions there are entirely new production mechanisms that are absent or inefficient in proton collisions. The strong electromagnetic fields in heavy-ion collisions can drastically increase the production cross section for some exotic states that couple to photons. This can be exploited by considering ultraperipheral heavy-ion collisions [25], as emphasized in recent publications on monopoles [26] and axionlike particles [27]. It has also been suggested that thermal processes in the quark-gluon plasma (QGP) can help one to produce a sizable number of exotic states [10].

This article presents an illustrative study with an analysis strategy based entirely on points (i) and (iii). The effect of point (ii) is model dependent, and explained in more detail in Sec. III. A detailed quantitative analysis of the effects deriving from point (ii) goes beyond the scope of the present article, whose main purpose is to point out the potential of heavy-ion collisions for LLP searches. We do not explore the (strongly model dependent) point (iv) in the present work. A list of references on this topic can, e.g., be found in Ref. [10].

\section{TRACK AND VERTEX MULTIPLICITIES}

Historically, heavy-ion collisions have been considered an overly complicated environment, therefore unsuitable for precise measurements of particle properties or searches of rare phenomena, because of their large final-state particle multiplicity, as opposed to $p p$ collisions. However, due to the high pileup during Run 4 in $p p$ collisions, the track multiplicity is expected to become comparable for $p p$ and $\mathrm{PbPb}$ collisions while it is smaller for lighter ion collisions [29].

In $\mathrm{PbPb}$ collisions, hard-scattering signals are more likely to originate in the most central events, where up to around 2000 charged particles are produced per unit of rapidity at $\sqrt{s_{\mathrm{XX}}}=5.52 \mathrm{TeV}$ [30], meaning that around 10000 tracks can be found in the tracking acceptance of the multipurpose experiments ATLAS and CMS. In contrast, $p p$ collisions during standard runs in 2017 were typically overlaid by about 30 pileup events, each adding about 25

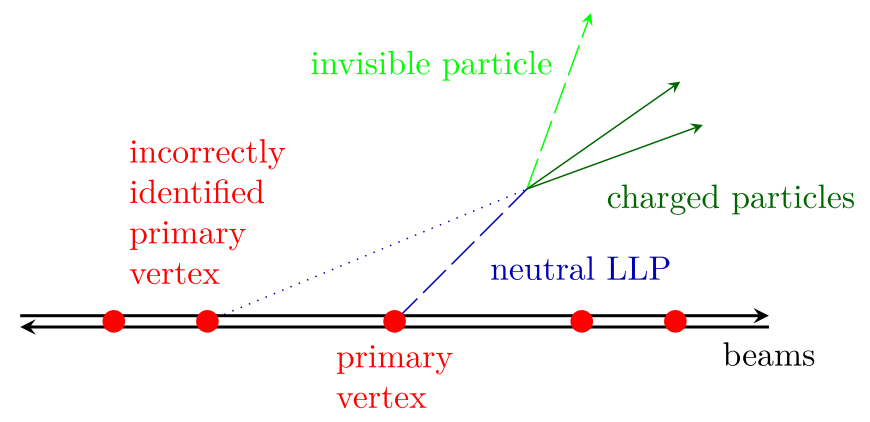

FIG. 1. Example of a signature that is difficult to search for in high pileup $p p$ collisions. Heavy-ion collisions can provide a cleaner environment. 
charged particles on average within the tracking acceptance of the multipurpose detectors [31-33], meaning that $\approx 750$ charged particles per event are coming from pileup. This is not expected to increase by a large factor until the end of Run 3. The HL-LHC will bring a big jump: current projections assume that, in order to accumulate $3000 \mathrm{fb}^{-1}$ as planned, each bunch crossing will be accompanied by about 200 pileup events [34,35], meaning 5000 additional charged particles per hard-scattering event. In conclusion, in the HL-LHC era the difference in track multiplicity between most-central $\mathrm{PbPb}$ and $p p$ collisions will reduce to a mere factor of 2 . A lot of ingenuity has been invested by the major LHC experiments in recent years to overcome the issues deriving from such a large track multiplicity $[36,37]$. In addition to the planned detector upgrades, all particle reconstruction and identification algorithms have been made more robust and optimized for a regime of very large multiplicities, and these efforts automatically benefit also the analysis of heavy-ion data.

Although a very large track multiplicity is expected to degrade the reconstruction and identification of displaced vertices, the adverse effect of pileup on vertex-finding performance is caused more by the presence of additional primary-interaction vertices than from the sheer number of tracks. This is, e.g., demonstrated by the comparison of $b$ tagging performance in $t \bar{t}$ studies in $p p, p \mathrm{~Pb}$, and $\mathrm{PbPb}$ collisions $[38,39]$. Using the same algorithm as in the standard $p p$ analysis, and requiring an equal efficiency of correctly tagged $b$-quark-initiated jets, the misidentification rate of light jets is smaller in $p \mathrm{~Pb}$ than in $p p$ events $(0.1 \%$ vs $0.8 \%$ ) in spite of the larger track multiplicity [38]. However, in the case of $\mathrm{PbPb}$ collisions a dedicated retuning of the $b$-tagging algorithm was necessary in order to recover a comparable efficiency. Nevertheless an acceptable purity versus efficiency (sufficient to provide evidence for top quark production) was achieved even for the most central collisions, in which the track multiplicity is maximal [39]. Similar qualitative considerations apply to the case of algorithms for the reconstruction of long-lived particles.

\section{AVERAGE INSTANTANEOUS LUMINOSITY}

The maximum luminosity achievable in heavy-ion collisions is constrained by multiple factors.

(1) Technical limits set on the injector performance.

(2) The total cross section per nucleon is increased compared to $p p$ collisions due to the additional sizable electromagnetic contributions. This results in a more rapid decline of the beam intensity. Moreover, most of the interactions are unwanted electromagnetic interactions caused by the stronger electromagnetic fields and soft hadronic processes, i.e., electromagnetic dissociation (EMD) and boundfree pair production (BFPP), see, e.g., Refs. [40-42] and references therein for details. The change of the mass/charge ratio caused by these processes leads to secondary beams that can potentially quench the LHC magnets. This problem was only recently mitigated for ATLAS and CMS by directing the secondary beams between magnets, while a special new collimator is required for ALICE [43,44].

(3) Collecting the maximum rate of events that the LHC can deliver is not necessarily ideal for all the experiments. For instance, the ALICE experiment is limited in the amount of data that it can acquire by the repetition time of its time projection chamber [45], thus instantaneous luminosity is leveled at their interaction point by adjusting the horizontal separation between the bunches. Similarly also the $\mathrm{LHCb}$ experiment only uses about $10 \%$ of the available beam intensity [46].

The upper limit on the achievable instantaneous luminosity depends on the charge $Z$ and mass $A$ of the accelerated nuclei in a complicated manner and is currently under investigation. For the purpose of the present article, we use the numbers presented in II, which are computed based on estimates presented at a recent HL-LHC workshop [47], see also [24]. In the following, we briefly summarize how we used these data. The instantaneous luminosity at one interaction point (IP) scales according to [48]

$$
\mathcal{L} \propto n_{b} N_{b}^{2},
$$

where $n_{b}$ is the number of bunches per beam and $N_{b}$ is the number of nucleons per bunch. The decay of the beam due to interactions follows

$$
\frac{\mathrm{d} N_{b}}{\mathrm{~d} t}=-\sigma_{\mathrm{tot}} \frac{n_{\mathrm{IP}}}{n_{b}} \mathcal{L}=-\frac{N_{b}^{2}}{N_{0} \tau_{b}},
$$

where $n_{\mathrm{IP}}$ is the number of interaction points, $\sigma_{\mathrm{tot}}$ is the total cross section, $N_{0}=N_{b}(0)$ is the initial number per bunch at beam injection, and

$$
\tau_{b}=\frac{n_{b}}{\sigma_{\text {tot }} n_{\mathrm{IP}}} \frac{N_{0}}{\mathcal{L}_{0}} \propto \frac{1}{\sigma_{\mathrm{tot}} n_{\mathrm{IP}} N_{0}}
$$

is the beam lifetime. Here $\mathcal{L}_{0}$ is the initial instantaneous luminosity at beam injection. Therefore, the number of nucleons per bunch decays according to

$$
N_{b}(t)=\frac{N_{0}}{1+\theta}, \quad \text { with } \quad \theta=\frac{t}{\tau_{b}} .
$$

The evolution of the instantaneous luminosity $\mathcal{L}(t)$ and integrated luminosity $L(t)$ are then

$$
\mathcal{L}(t)=\frac{\mathcal{L}_{0}}{(1+\theta)^{2}}, \quad L(t)=\mathcal{L}_{0} \tau_{b} \frac{\theta}{1+\theta} .
$$

The turnaround time $t_{\mathrm{ta}}$ is the average time between two physics runs. Therefore, the average luminosity is 


$$
\mathcal{L}_{\text {ave }}(t)=\frac{L(t)}{t+t_{\mathrm{ta}}}
$$

which is maximized for

$$
t_{\mathrm{opt}}=\tau_{b} \sqrt{\theta_{\mathrm{ta}}}, \quad \text { with } \quad \theta_{\mathrm{ta}}=\frac{t_{\mathrm{ta}}}{\tau_{b}} .
$$

Finally, the average luminosity for the optimal runtime is

$$
\mathcal{L}_{\text {ave }}\left(t_{\text {opt }}\right)=\frac{\mathcal{L}_{0}}{\left(1+\sqrt{\theta_{\text {ta }}}\right)^{2}}
$$

Additionally, the initial bunch intensity follows roughly

$$
N_{b}\left({ }_{Z}^{A} \mathrm{X}\right)=N_{b}\left({ }_{82}^{208} \mathrm{~Pb}\right)\left(\frac{Z}{82}\right)^{-p},
$$

where the exponent characterizes the number of nucleons per bunch. For a given isotope, it is limited by the heavyion injector chain, the bunch charges, and intrabeam scatterings. Simple estimates based on fixed target studies with Ar beams suggest that $1 \lesssim p \lesssim 1.9$ is realistic [47].

\section{AN EXAMPLE: HEAVY NEUTRINOS}

In the following, we use the example of heavy neutrinos with masses below the electroweak scale that interact with the SM exclusively through their mixing with ordinary neutrinos, to illustrate the potential of new physics searches in heavy-ion collisions. This is an extremely conservative approach for two reasons. First, we do not take advantage of any of the new production mechanisms that the strong electromagnetic fields or the QGP offer in comparison to proton collisions, see point (iv). Second, we do not take advantage of the lack of pileup, point (ii), which we do not expect to play a major role in the minimal seesaw model considered here. This point can, however, give heavy-ion collisions a crucial advantage over proton runs in searches for signatures with a more complicated topology than the decays shown in Fig. 2. In the context of heavy neutrinos this could, e.g., be the case in left-right symmetric models [49-51] where decays mediated by Majorons can lead to pairs of displaced vertices [52].

Right-handed neutrinos $\nu_{R}$ appear in many extensions of the SM. The implications of their existence strongly depend on the values of their masses. The $\nu_{R}$ could solve several open puzzles in cosmology and particle physics, see, e.g., [53] for a review. Most notably they can explain the light neutrino masses via the type-I seesaw mechanism [54-59], which requires one flavor of $\nu_{R}$ for each nonzero neutrino mass in the SM. In addition they may explain the baryon asymmetry of the Universe via leptogenesis [60], act as dark matter (a) Electroweak production.

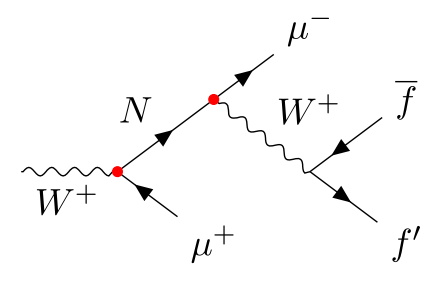

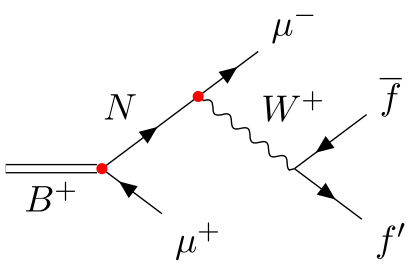

(b) B-meson production.
FIG. 2. Production and decay of heavy neutrino mass eigenstates $N$ via (a) $W$ bosons and (b) $B$ mesons. The tiny couplings inducing the displaced signature are indicated by red vertices.

candidates [61], address various anomalies observed in neutrino oscillation experiments [62], or generate the Higgs potential radiatively [63]. ${ }^{1}$

Since right-handed neutrinos are gauge singlets, the number of their flavors is not constrained to be equal to the SM generations by anomaly considerations. At least two flavors of $\nu_{R}$ are required to explain the observed neutrino oscillation data via the seesaw mechanism. Here we work in a simple toy model with only a single flavor of $\nu_{R}$ with mass $M$, which is sufficient because the displaced vertex signature does not rely on interference effects among different neutrinos or correlations between their parameters. The minimal extension of the SM with right-handed neutrinos can be obtained by adding all renormalizable operators that only contain $\nu_{R}$ and SM fields to the SM Lagrangian,

$\mathcal{L}_{\nu_{R}}=\frac{\mathrm{i}}{2} \overline{\nu_{R}} \not \nu_{R}-F_{a} \overline{\ell_{L a}} \varepsilon \phi^{*} \nu_{R}-\frac{1}{2} \overline{\nu_{R}^{c}} M \nu_{R}+$ H.c.

Here $\phi$ is the SM Higgs doublet, $\ell_{L a}$ are the SM lepton doublets, the $F_{a}$ are the Yukawa coupling constants to the $\mathrm{SM}$ lepton generation $a$, and $\varepsilon$ is the antisymmetric $\mathrm{SU}(2)$ tensor.

The heavy neutrino interactions with the SM can be described by the mixing angles $\theta_{a}=v F_{a} / M$ with $v=\langle\phi\rangle \simeq 174 \mathrm{GeV}$, which characterize the relative suppression of their weak interactions compared to those of the light neutrinos. The Lagrangian that describes the interaction of the heavy neutrino mass eigenstate $N \simeq \nu_{R}+$ $\theta_{a} \nu_{L a}^{c}+$ c.c. with the SM reads

$$
\begin{aligned}
\mathcal{L} \supset & -\frac{m_{W}}{v} \bar{N} \theta_{a}^{*} \gamma^{\mu} e_{L a} W_{\mu}^{+}-\frac{m_{Z}}{\sqrt{2} v} \bar{N} \theta_{a}^{*} \gamma^{\mu} \nu_{L a} Z_{\mu} \\
& -\frac{M}{v} \theta_{a} h \overline{\nu_{L} \alpha} N+\text { H.c. }
\end{aligned}
$$

where $h$ is the physical Higgs field after spontaneous breaking of the electroweak symmetry. The mixing angles

\footnotetext{
${ }^{1}$ For further details we refer the reader to the following reviews on the matter-antimatter asymmetry [64], the perspectives to test leptogenesis [65], sterile neutrino dark matter [66,67] and experimental searches for heavy neutrinos [68-71].
} 


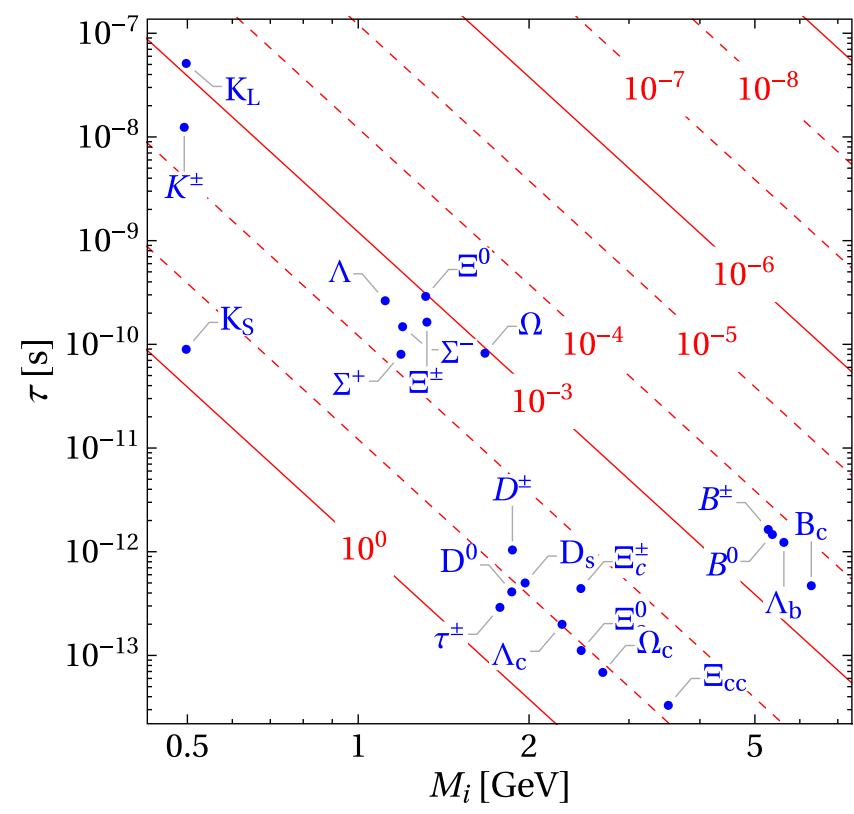

FIG. 3. Heavy neutrino mixing $U^{2}$ (red lines) compared to potentially relevant SM backgrounds (blue dots) as a function of particle mass $M_{i}$ and lifetime $\tau$. Figure taken from Ref. [76]. In addition to these SM backgrounds, secondary nuclear interactions in the detector material constitute a source of background for displaced signatures. For comparable track multiplicity the effect of the SM backgrounds would, depending on the particle's lifetime, be equal or worse in $p p$ data compared to heavy-ion data.

$U_{a}^{2} \equiv\left|\theta_{a}\right|^{2}$ can be large enough to produce sizable numbers of heavy neutrinos in collider experiments if the heavy neutrinos approximately respect a generalized B-L symmetry [72-74], where B and L denote baryon and lepton number, respectively (see also [75]).

For $M$ below the weak gauge boson masses, the heavy neutrinos can be long-lived enough to produce displaced vertex signals at the LHC [18,76-93] or at a future collider $[71,94-97],{ }^{2}$ see Fig. 3. In this mass range the Lagrangian (10) effectively describes the phenomenology of the Neutrino Minimal Standard Model ( 2 MSM) [105,106], a minimal extension of the SM that can simultaneously explain the light neutrino masses, dark matter, and the baryon asymmetry of the Universe [107,108], see [109] for a review. The dominant production channel for $M>5 \mathrm{GeV}$ is the decay of real $Z(W)$ bosons, in which the heavy neutrinos are produced along with a neutrino $\nu_{a}$ (charged lepton $\ell_{a}$ ), while for $M<5 \mathrm{GeV}$ the production in $b$-flavored hadron decays dominates, see Fig. 2. The number of heavy neutrinos that are produced along with a lepton of flavor

\footnotetext{
${ }^{2}$ Such searches could be much more sensitive in models where the heavy neutrinos have additional interactions [52,98-104]. Heavy-ion collisions can be a promising place to search for signatures with two displaced vertices, see, e.g., [52], that would benefit from better vertex identification, see point (ii).
}

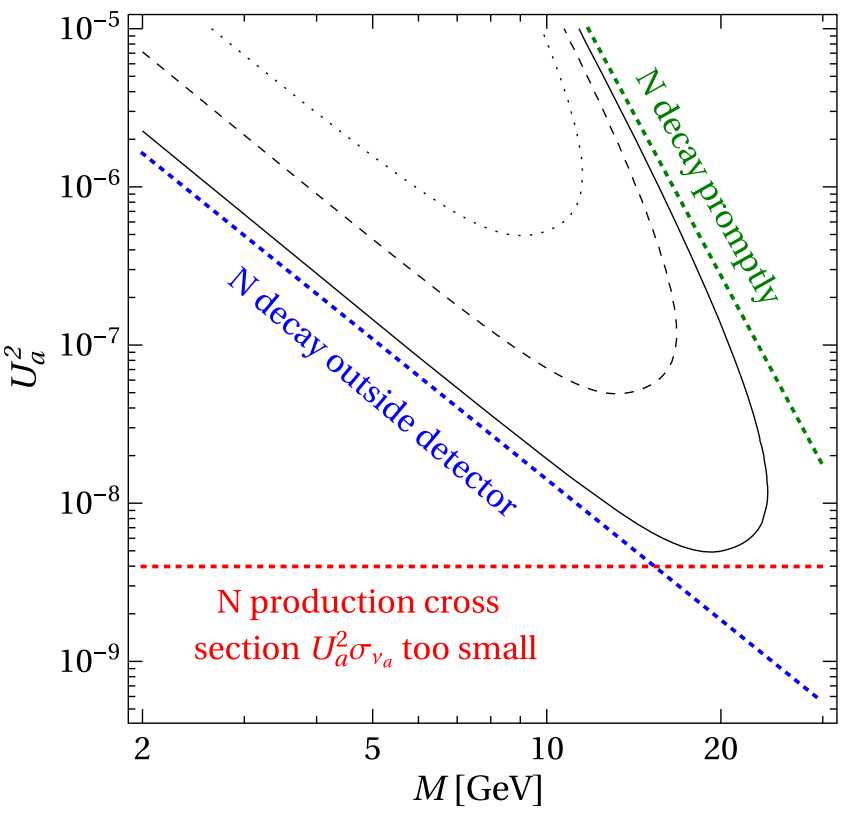

FIG. 4. We show a sensitivity estimate for $W$ induced $N$ decays in $p p$ collisions based on the simplified detector model (12) with $l_{0}=5 \mathrm{~mm}$ and $l_{1}=20 \mathrm{~cm}$. The three sensitivity curves (black) correspond to nine expected events with integrated luminosities of $3,30,300 \mathrm{fb}^{-1}$ as a function of heavy neutrino mass $M$ and mixing $U_{a}^{2}$. The colored dotted lines illustrate the three main obstacles in improving the reach.

$a$ can be estimated as $\sim L \sigma_{\nu} U_{a}^{2}$, where $\sigma_{\nu}$ is the production cross section for light neutrinos. It is roughly given by $\sigma_{\nu} \simeq$ $\sigma_{W} / 3$ in $W$ decays and $\sigma_{\nu} \simeq \sigma_{B} / 9$ in $B$ decays, where $\sigma_{W}$ and $\sigma_{B}$ are the $W$ and $B$ production cross sections in a given process. They then decay semileptonically or purely leptonically, see, e.g., [68,108,110-113].

The number of displaced vertex events with a lepton of flavor $a$ at the first vertex and a lepton of flavor $b$ from the second vertex that can be seen in a detector can then be estimated as

$N_{\text {obs }} \simeq L \sigma_{\nu} \frac{U_{a}^{2} U_{b}^{2}}{U^{2}}\left[\exp \left(-\frac{l_{0}}{\lambda_{N}}\right)-\exp \left(-\frac{l_{1}}{\lambda_{N}}\right)\right] f_{\text {cut }}$.

Here $l_{1}$ is the length of the effective detector volume in a simplified model of a spherical detector, $l_{0}$ the minimal displacement that is required by the trigger, $\lambda_{N}=\beta \gamma / \Gamma_{N}$ is the particle decay length, where $\Gamma_{N}$ is the heavy neutrino decay width, $\beta$ is the heavy neutrino velocity, and $\beta \gamma=$ $|\boldsymbol{p}| / M$ the usual Lorentz factor, $U^{2}=\sum_{a} U_{a}^{2}$ is the total mixing, and $f_{\text {cut }} \in[0,1]$ is an overall efficiency factor that parametrizes the effects of cuts due to triggers, deviations of the detector geometry from a sphere, and detector efficiencies. The analytic formula (12) allows for an intuitive understanding of the sensitivity curves obtained from simulations, see Fig. 4. As illustrated in Fig. 8, it can reproduce the results of simulated data surprisingly well. 
One may wonder whether the heavy neutrinos can leave the dense plasma that surrounds the collision point. Intuitively, this should clearly be the case because the scattering cross section of heavy neutrinos is suppressed by a factor $\sim U^{2}$ compared to that of ordinary neutrinos. For a more quantitative estimate, we can evaluate the mean free path $\lambda_{T}$ of the relativistic heavy neutrinos of energy $\omega_{p} \simeq m_{W} / 2$ that are produced in real gauge boson decays as $\lambda_{T} \simeq \gamma \beta / \Gamma_{T}$, where $\Gamma_{T}$ is the thermal damping rate in a plasma of temperature $T$. In this regime it is known that $\Gamma_{T}<U^{2} T^{2} / \omega_{p}$ [122]. We can therefore estimate

$\lambda_{T} \simeq \frac{\gamma \beta}{\Gamma_{T}}>\frac{|\boldsymbol{p}| \omega_{p}}{U^{2} T^{2} M}>\frac{|\boldsymbol{p}|^{2}}{U^{2} T^{2} M}>\frac{1}{U^{2} M} \approx \frac{0.2 \mathrm{GeV}}{U^{2} M} \mathrm{fm}$,

which is orders of magnitude larger than a few tens of femtometers.

Since $f_{\text {cut }}$ is largest for muons, in the following we concentrate on a benchmark model in which the heavy neutrinos mix exclusively with the second generation $\left(U^{2}=U_{\mu}^{2}\right){ }^{3}$ The expression (12) then further reduces to

$$
N_{\text {obs }} \simeq L \sigma_{\nu} U_{\mu}^{2}\left[\exp \left(-\frac{l_{0}}{\lambda_{N}}\right)-\exp \left(-\frac{l_{1}}{\lambda_{N}}\right)\right] f_{\text {cut }}
$$

\section{A. Heavy neutrinos from $W$-boson decay}

\section{Event generation}

We first study the perspectives to find heavy neutrinos produced in the decay of $W$ bosons in a displaced vertex search. Our treatment of the detector closely follows that of Ref. [76], but we have adapted the simulation of the production for different colliding isotopes. We calculate the Feynman rules for Lagrangian (11) with FeynRules 2.3 [124], using the implementation [125] that is based on the computations in Refs. $[68,126]$. Then we generate events for the processes shown in Fig. 2 with MadGraph5_aMC@NLO 2.6.4 [28], which is capable of generating events for heavyion collisions if provided with the appropriate parton distribution functions (PDFs). For the simulation of lead collisions we use published PDFs [127]. However, for argon and other intermediate ions there are no published PDFs, therefore we calculate the ion PDFs by scaling the proton PDFs. The PDF for a quark of flavor $a$ within an ion with mass number $A=p+n$ is denoted by $f_{p, n}^{a}\left(x, Q^{2}\right)$, where $x$ is the Bjorken fraction defined as the ratio of the

\footnotetext{
${ }^{3}$ Realistic flavor mixing patterns in the seesaw model in view of current neutrino oscillation data have recently been studied in [123], we refer the interested reader to this article and references therein.
}

parton energy over the ion energy. It can be approximated by a rescaling of the proton PDF $f_{1,0}$ via

$$
\begin{gathered}
f_{p, n}^{d}(x)=p f_{1,0}^{d}(A x)+n f_{1,0}^{u}(A x), \\
f_{p, n}^{u}(x)=p f_{1,0}^{u}(A x)+n f_{1,0}^{d}(A x), \\
f_{p, n}^{\bar{d}}(x)=p f_{1,0}^{\bar{d}}(A x)+n f_{1,0}^{\bar{u}}(A x), \\
f_{p, n}^{\bar{u}}(x)=p f_{1,0}^{\bar{u}}(A x)+n f_{1,0}^{\bar{d}}(A x), \\
f_{p, n}^{f}(x)=A f_{1,0}^{f}(A x),
\end{gathered}
$$

where the index $f$ denotes quarks beyond the first generation and gluons. For the sake of notational clarity we have dropped the scale dependence $Q^{2}$.

We find that the effects of the nuclear PDFs can be neglected after comparing them to other sources of uncertainty in our analysis. We use MadWidth [128] to calculate the $N$ decay width, ${ }^{4}$ the resulting lifetime is given in Fig. 3. Subsequently, we simulate the decays with MadSpin $[129,130]$. Finally, we hadronize the colored particles and generate hadronic showers with PYTHIA 8.2 [131]. We calculate the detector efficiencies of the CMS detector using our own code based on public information of the detector geometry. Most importantly, we use a pseudorapidity coverage of $|\eta| \leq 4$ and use for the extension of the tracker 1.1 and $2.8 \mathrm{~m}$ in the transversal and longitudinal direction, respectively [37]. In [76] it has been shown that in $p p$ collisions the expected performance of the ATLAS detector is comparable to the one of the CMS detector for this search strategy. We expect the same to be true in heavyion collisions.

We search this signal in event samples that have either been triggered by a single muon or by a pair of muons. The minimal transverse momentum $p_{T}$ of the muon used for the pair triggers can be softer than in the single muon triggers. For the tagging and tracking efficiencies we use the CMS detector card values of DELPHES 3.4.1 [132]. In order to reduce the background from long-lived SM hadrons we require that the secondary vertices have a minimal displacement $l_{0}$ of $5 \mathrm{~mm}$. In order to suppress further backgrounds, in particular from nuclear interactions of hadrons produced in the primary collisions with the detector material, we require at least two displaced tracks with an invariant mass of at least $5 \mathrm{GeV}$ in the reconstruction of the displaced vertices, see Ref. [83]. The reconstruction efficiency is near $100 \%$ if the produced particles traverse the entire tracker. If a particle traverses only a fraction of the tracker the efficiency is reduced. We adapt a ray tracing

\footnotetext{
${ }^{4}$ Madwidth uses quarks instead of hadrons in the final states. The resulting error is relatively small as long as all particles are relativistic, as we explicitly checked by comparison with the results in [110].
} 
$[133,134]$ method to compute the particle's trajectory and use the length of the remaining path within the tracking system as the criterion to estimate the vertex reconstruction efficiency. It has recently been shown in Ref. [135] that the detection efficiency drops only linearly with the displacement if advanced algorithms are used. We adopt this functional dependence and assume that the maximal displacement that can still be detected can be improved by a factor 2 if optimized algorithms are used. This strategy closely follows the approach taken in Ref. [76].

We fix the integrated luminosity in $\mathrm{PbPb}$ runs to $5 \mathrm{nb}^{-1}$, a realistic value for one month in the heavy-ion program. We then use the relations presented in Sec. IV to estimate the integrated luminosity that could be achieved with Ar in the same period as 0.5 and $5 \mathrm{pb}^{-1}$ for pessimistic and optimistic assumptions for the scaling behavior, respectively. For protons we use $50 \mathrm{fb}^{-1}$.

\section{Backgrounds}

Following the approach in Ref. [76], we work under the assumption that the SM background can be efficiently excluded by the cuts on the invariant mass and the displacement, see Fig. 3. Quantifying the remaining backgrounds would require a very realistic simulation of the whole detector. These include cosmic rays and beam-halo muons, which only occur at a low rate in the experimental caverns and can mostly be recognized [136], as well as scattering of SM neutrinos from the collision point with the detector, which have a low cross section of charged-current interaction in the detector material. In summary, we assume that the background number is smaller than one and do an (under this assumption) conservative statistical analysis with one background event, using the nonobservation of four events and the observation of nine events for exclusion and discovery, respectively.

\section{Results}

We present our results in Fig. 5. It shows that the suppression of the number of events due to the reduced instantaneous luminosity of heavy-ion runs compared to proton runs overcompensates for the $A^{2}$ enhancement per collision, i.e., point (i), so that $\mathrm{Pb}$ collisions are clearly not competitive. For lighter nuclei like Ar the perspectives are somewhat better, as the expected number of events per unit of running time is only about an order of magnitude smaller than in proton runs. If the heavy neutrinos have mixing angles slightly below the current experimental limits, then they would first be discovered in proton collisions, but heavy-ion collisions would still offer a way to probe the interactions of the new particles in a very different environment. For heavy neutrinos that are produced in $W$-boson decays, the sensitivity is only marginally increased when lowering trigger thresholds, i.e., point (iii), because most $\mu^{ \pm}$from the primary vertex have
$p_{T}>25 \mathrm{GeV}$ due to the mass of the $W$ boson. It remains well below what can be achieved in proton collisions at the LHC [76,78-81,83-92].

\section{B. Heavy neutrinos from $B$-meson decays}

The situation is very different for heavy neutrinos produced in $B$-meson decays. The cutoff in sensitivity along the $M$ axis in this case is not determined by the fact that the $N$ decays too quickly to give a displaced vertex signal, but by kinematics: the production cross section exhibits a sharp cut when $M$ approaches the $B$-meson mass $m_{B}$. Since this cut occurs in a mass range where the expression (12) suggests that the sensitivity should still improve when increasing $M$, see Fig. 4, we expect that one can achieve maximal sensitivity just below the threshold. This means that the sensitivity is maximal in a region where the momenta in the $B$-meson rest frame of both the $N$ and the $\mu^{ \pm}$that is produced along with it are much smaller than $m_{B}$. The $p_{T}$ distribution of $B$ mesons in the laboratory frame peaks around $3 \mathrm{GeV}$, see Fig. 6 (a). As a result, the vast majority of $\mu^{ \pm}$have $p_{T}$ well below standard $p_{T}$ cuts. Hence, there is enormous potential for improving the sensitivity if one can lower the trigger thresholds on the primary muon $p_{T}$. For $B$-meson induced processes in heavy-ion collisions we assume a trigger threshold of $3 \mathrm{GeV}$, which roughly corresponds to the kinematic limits dictated by the magnetic bending and the geometry of tracking detectors.

The production of heavy neutrinos in $B$-meson decays cannot be simulated in the same way as described in Sec. VA. A detailed simulation of $N$ production from $B$ mesons and their decay is technically challenging and goes beyond the scope of this work, the main purpose of which is to estimate the order of magnitude of the sensitivity that can be reached in heavy-ion runs. Therefore, we resort to a modification of the simplified detector model (12) to determine the number of events. If the masses of all final-state particles were negligible, we could express $\sigma_{\nu}=\sigma_{B} / 9$, where $\sigma_{B}$ is the total $B$-meson production cross section and the factor $1 / 9$ accounts for the branching ratio of the decay into final states including neutrinos. There is a wide range of SM two and three body decays into neutrinos, in all of which the SM neutrino could be replaced by an $N$. In the two body decay $B^{ \pm} \rightarrow \mu^{ \pm} N$ the heavy neutrino mass can be taken into account by multiplying by a simple phase space factor,

$N_{\mathrm{obs}}=\frac{L \sigma_{B}}{9}\left(1-\frac{M^{2}}{m_{B}^{2}}\right)^{2} U_{\mu}^{2}\left(e^{-l_{0} / \lambda_{N}}-e^{-l_{1} / \lambda_{N}}\right) f_{\mathrm{cut}}$.

While this decay is helicity suppressed in the SM, this is not the case for the decay into heavy neutrinos. 


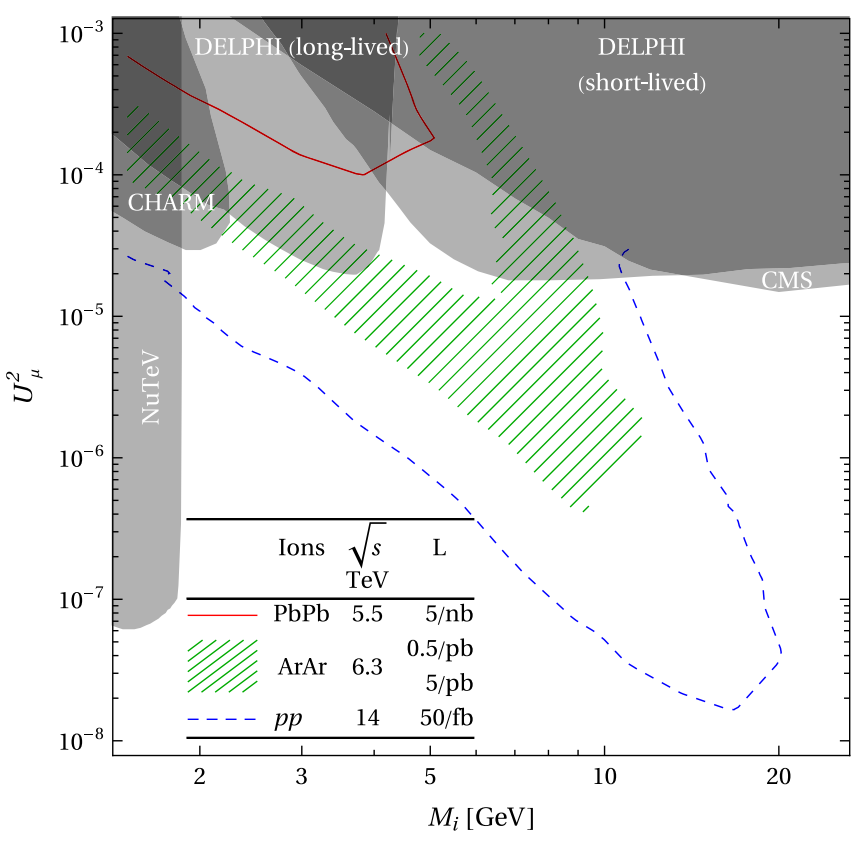

(a) Exclusion with $2 \sigma$.

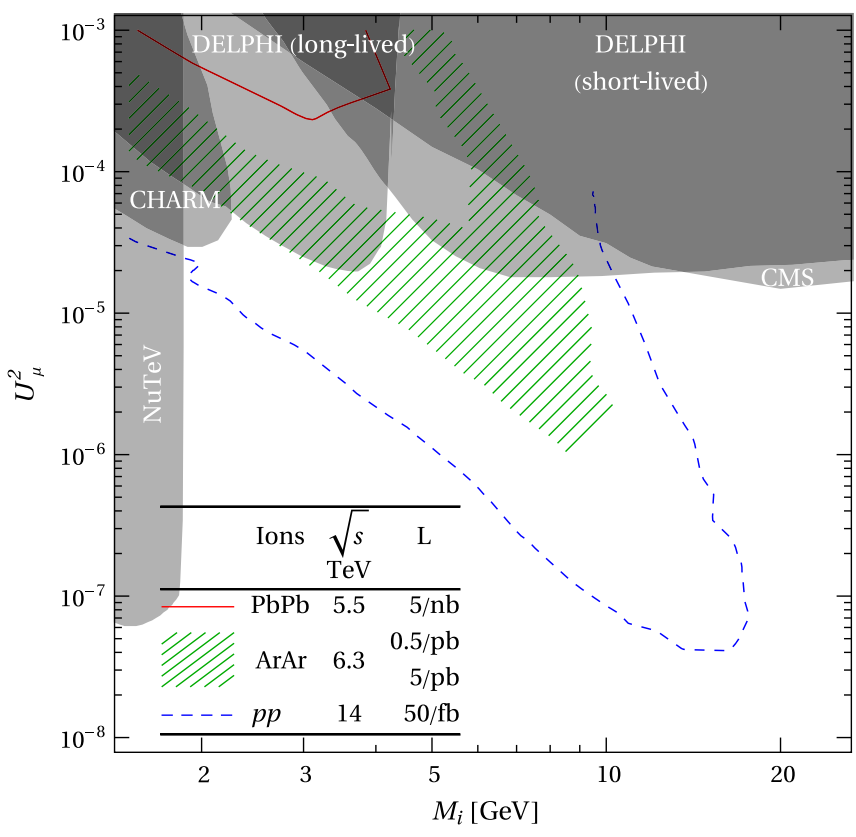

(b) Discovery with $5 \sigma$.

FIG. 5. Sensitivity of the CMS detector for heavy neutrinos produced in $W$ decays, as a function of the heavy neutrino mass $M$ and mixing $U_{\mu}^{2}$, in $\mathrm{PbPb}$ (solid red lines), ArAr (green hashed band), and $p p$ (dashed blue lines) collisions at 5.5, 6.3, and 14 $\mathrm{TeV}$, respectively. The luminosities are indicated in the plot and roughly correspond to equal running times of one month. (a) and (b) correspond to exclusion (four events) and discovery (nine events), respectively. We expect a comparable result for the ATLAS detector. The results are based on a simulation of $W$ induced processes using MadGraph5_aMC@NLO with the parameters described in Sec. VA. The green band reflects the current uncertainty in the beam intensity that can be achieved in ArAr collisions. The gray areas represent the exclusion limits of former experiments NuTeV [114], CHARM [115], DELPHI [116], and CMS [117]. We do not display a constraint on the mixing angle from the requirement to generate the light neutrino masses because light neutrino oscillation data only imposes a lower bound on the mixing of an individual heavy neutrino species if one makes additional model-dependent assumptions [118].

\section{Matching the simplified detector model to simulations}

We determine the parameters $l_{0}, l_{1}$, and $f_{\text {cut }}$ in the model (16) by fitting the simplified detector model (12) to the results of our simulations for $N$ production in $W$ decays shown in Fig. 5. This corresponds to modeling the LHC detectors ATLAS or CMS as spherical, which turns out to be a good estimate up to factors of 2 to 3 , see Fig. 8. For the neutrino production cross section $\sigma_{\nu}$ in $W$-boson decays we use the results from MadGraph5_aMC@NLO, i.e., $1.12 \times$ $10^{4} \mathrm{pb}$ for proton collisions at $14 \mathrm{TeV}$ and $40^{2} \times$ $4898 \mathrm{pb}$ and $208^{2} \times 4228 \mathrm{pb}$ for $\mathrm{Ar}$ at $6.3 \mathrm{TeV}$ and $\mathrm{Pb}$ at $5.5 \mathrm{TeV}$, respectively.

In order to account for the Lorentz factor $\beta \gamma$ for each choice of $M$, we compute the $N$ momentum in the laboratory frame as a function of the $W$-boson momentum and the angle between the spacial $W$ and $N$ momenta. We then average Eq. (12) over $W$ momenta, using a distribution which we have generated simulating the process $p p \rightarrow W$ with up to two jets using MadGraph5_aMC@NLO with subsequent hadronization and matching with soft jets via PYTHIA. With $l_{0}=2 \mathrm{~cm}$ and $l_{1}=20 \mathrm{~cm}$ we can reproduce the results of our simulation shown in 5 in good approximation if we set the overall effective efficiency to $f_{\text {cut }}=0.1$.

The fitted parameter values can be understood in terms of physical arguments. The choice $l_{0}=2 \mathrm{~cm}$ is qualitatively in good agreement with what one would expect from the geometrical cuts 0.5 and $10 \mathrm{~cm}$ on the minimal displacement in transversal and longitudinal direction that were used in the simulation. $l_{1}=20 \mathrm{~cm}$ indicates a typical distance at which one can still reconstruct the displaced vertex. In the simulation we assumed that the vertex reconstruction efficiency linearly drops from $100 \%$ to zero between a displacement of $5 \mathrm{~mm}$ and $55 \mathrm{~cm}$, hence $20 \mathrm{~cm}$ is a reasonable value. The fact that all of the parameter values can be understood physically provides a strong self-consistency check for our approach. In Fig. 8 we show the ratio between the simplified detector model (12) and the results of the simulation described in Sec. VA 1 within the region where Eq. (12) predicts more than 0.1 events. Given the nonlinear dependence of the function (12) on the parameters and the fact that $N_{\text {obs }}$ changes over 6 orders of magnitude within this region, it is absolutely 


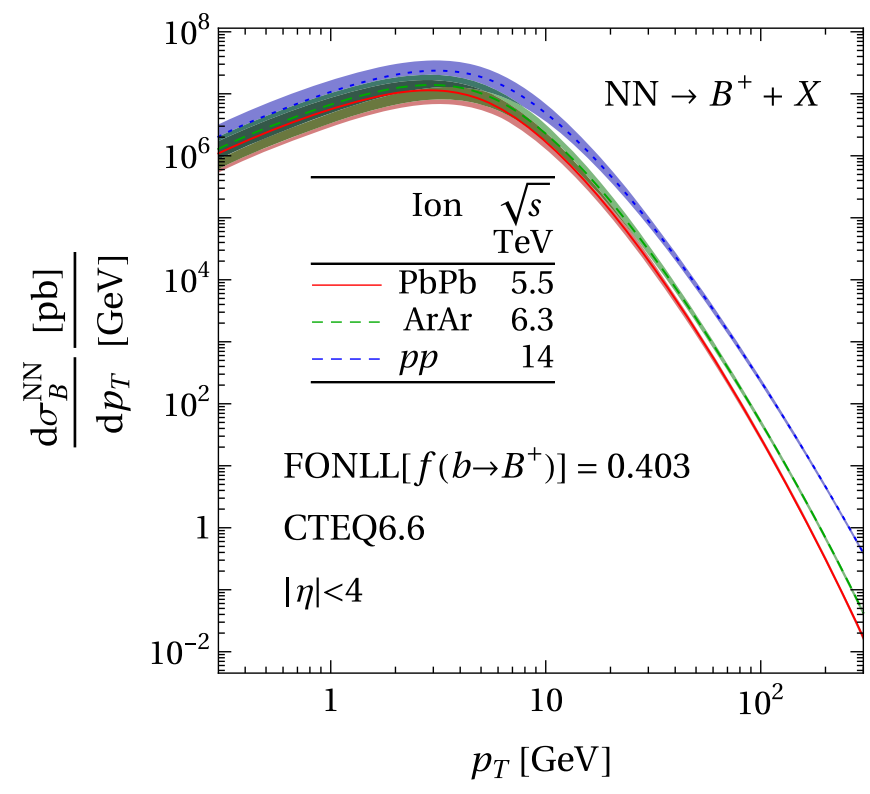

(a) Differential cross section $\mathrm{d} \sigma_{B}^{\mathrm{XX}} / \mathrm{d} p_{T}$ of $B$-mesons.

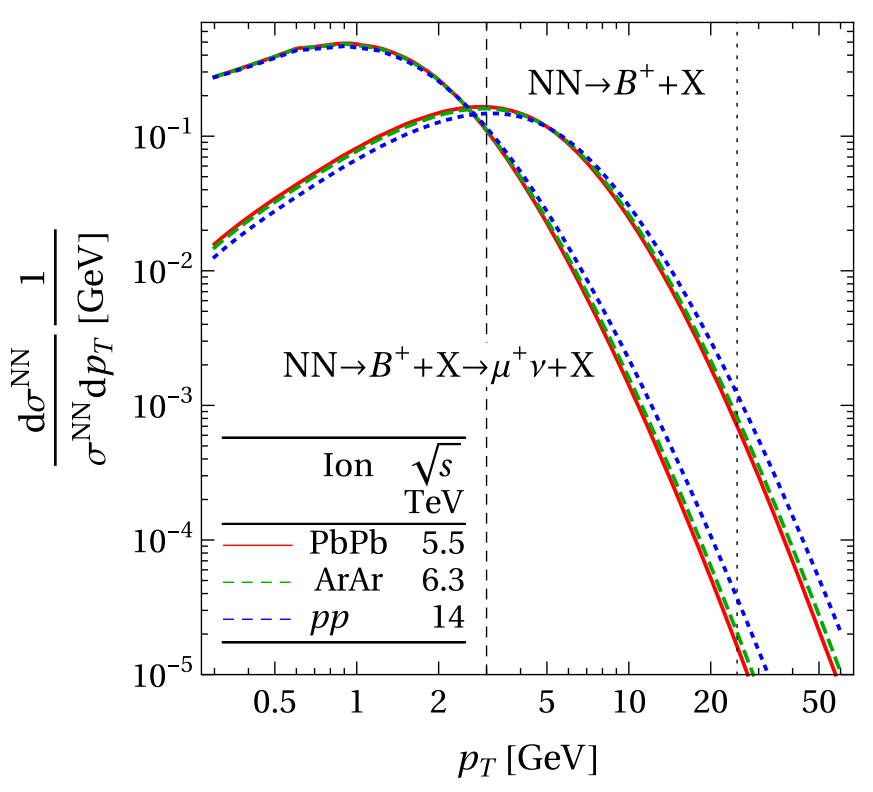

(b) Comparison of normalized differential cross sections $\mathrm{d} \sigma^{\mathrm{XX}} / \mathrm{d} p_{T} \sigma^{\mathrm{XX}}$.

FIG. 6. (a) Parton level differential cross section $\mathrm{d} \sigma_{B}^{\mathrm{XX}} / \mathrm{d} p_{T}$ of $B$ mesons per nucleon (including theoretical uncertainties) expected in collisions of the ions indicated in the plot. (b) Comparison between the differential cross sections of the $B$ mesons and those of the leading muons produced in their decay, each normalized to the respective integrated cross section. The estimates are based on proton collisions with the collision energy per nucleon $\sqrt{s}$ in heavy-ion collisions $14 \mathrm{TeV}$ (dotted blue lines) for $p p, 7 \mathrm{TeV}$ (dashed green lines) for $\mathrm{ArAr}$, and 5.5 TeV (solid red lines) for $\mathrm{PbPb}$. As shown in Fig. 7 the nuclear modification effects in good approximation only lead to an overall rescaling of the cross section, which has little effect on the comparison of event numbers for different $p_{T}$ cuts described in Sec. V B 2. All processes correspond to central collisions with $|\eta|<4$. The predictions have been derived with the FONLL framework [21-23] at NLO + NLL, using for the $b$-quark fragmentation fraction a value of $f\left(b \rightarrow B^{+}\right)=0.403$ [119] and the CTEQ 6.6 NLO parton distribution functions [120]. The dotted and dashed vertical lines at 25 and $3 \mathrm{GeV}$ correspond to the single muon trigger thresholds in proton and heavy-ion collisions, respectively.

nontrivial that the simplified model reproduces the simulation up to a factor 2 to 3 within that region.

\section{Computing the number of events}

In order to determine $\sigma_{B}$ in the model (16) we first compute the differential cross section $\mathrm{d} \sigma_{B} / \mathrm{d} p_{T}$ for $B$ mesons produced at different collision energies at NLO and next-to-leading log (NLL) within the FONLL framework [21-23], in the range $p_{T} \in[0,300] \mathrm{GeV}$ using $f\left(b \rightarrow B^{+}\right)=0.403$ for the $b$-quark fragmentation fraction [119] and the CTEQ 6.6 NLO parton distribution functions [120], accepting events with a pseudorapidity $|\eta|<4$. The results are shown in Fig. 6(a). We validate the predictions against experimental results [121], noticing that the data are mostly centered on the upper side of the theoretical uncertainty band, see Fig. 7. By using central value predictions we are thus underestimating the differential cross section, and the derived results can be interpreted as being conservative.

We fix the value of $\sigma_{B}$ by integrating over $\mathrm{d} \sigma_{B} / \mathrm{d} p_{T}=$ $A^{2} \mathrm{~d} \sigma_{B}^{\mathrm{XX}} / \mathrm{d} p_{T}$, where the integration limits have to be fixed by the $p_{T}$ cuts. We can incorporate the lower $p_{T}$ cut in heavy-ion collisions compared to proton collisions, point (iii), by computing $\sigma_{B}$ as an integral over $\mathrm{d} \sigma_{B} / \mathrm{d} p_{T}$ with different lower integration limits that reflect the different $p_{T}$ cuts on the primary muon. The $p_{T}$ distribution of the primary muons depends on $M$ and should be determined in a simulation. We take a much simpler approach that gives a very conservative estimate of the discovery potential in heavy-ion collisions. The $B$-meson $p_{T}$ distribution is a good proxy for the $p_{T}$ distribution of the leading muon if the heavy neutrino has a mass comparable to the $B$ meson, since in this case the muon will be soft in the $B$-meson rest frame. For smaller $M$ the two distributions can differ considerably due to the muon momentum in the $B$ rest frame. The modification is most extreme for $M=0$, in which case the kinematics is the same as if the $N$ in the final state is replaced by a SM neutrino. One can thus determine the muon $p_{T}$ distribution in the extreme cases $M=m_{B}$ and $M=0$ by computing the $p_{T}$ distribution of the $B$ mesons themselves as well as that of muons produced in their decay $B^{+} \rightarrow$ $\mu^{+} \nu_{\mu} X$, as shown in Fig. 6(b). In the second case the distribution peaks at considerably lower $p_{T}$. 


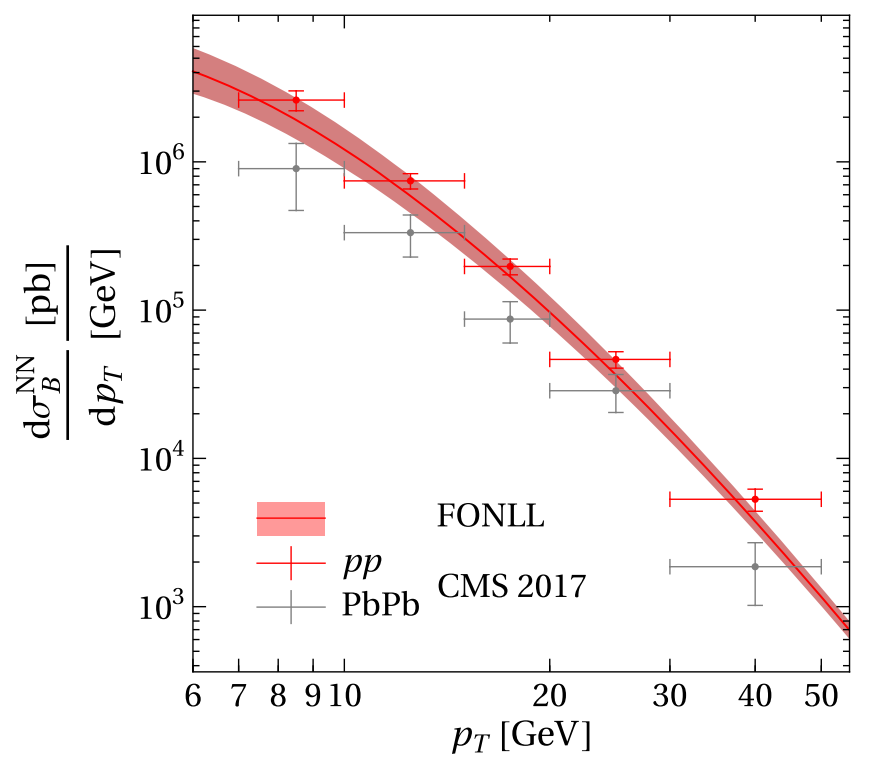

FIG. 7. Comparison between the theoretical differential production cross sections for $p p \rightarrow B^{+}+X$ at $\sqrt{s}=5.03 \mathrm{TeV}$ using $|\eta|<2.5$ computed with FONLL [21-23] as described in the caption of Fig. 6 (red band) and the experimental measurements for proton and lead collisions at $\sqrt{s}=5.02 \mathrm{TeV}$ (red and gray data points, respectively) [121]. The difference of $1 \mathrm{GeV}$ between the experimental and the theoretical center-of-mass energy is caused by a technical limitation of FONLL and the resulting uncertainty is negligible in comparison to the theoretical and experimental errors.

To keep the analysis simple and conservative, we directly apply the experimental $p_{T}$ cut on the leading muon to the $p_{T}$ distribution of the $B$ meson when computing $\sigma_{B}$ from $\mathrm{d} \sigma_{B} / \mathrm{d} p_{T}$. For $\sigma_{B}$ in proton collisions we use $25 \mathrm{GeV}<p_{T}<300 \mathrm{GeV}$, in heavy-ions collisions we use $3 \mathrm{GeV}<p_{T}<300 \mathrm{GeV}$. $p_{T}$ values below $3 \mathrm{GeV}$ are very hard to access even in heavy-ion collisions because the CMS magnetic field prevents particles with such low momentum from reaching the detector in most of the solid angle range where it is sensitive. ${ }^{5}$ If we had applied the same cuts to the muon distribution in $B^{+} \rightarrow \mu^{+} \nu_{\mu} X$ decays the predicted number of events in heavy-ion collisions would improve by more than an order of magnitude compared to our conservative estimate. The actual value of $\sigma_{B}$ lies in between these extreme cases. All other cuts and efficiencies are summarized in $f_{\text {cut }}$ and should be similar for proton and heavy-ion collisions, except for a

\footnotetext{
${ }^{5}$ In principle one should consider an $\eta$-dependent $p_{T}$ threshold. Realistic numbers for CMS in the same heavy-ion environment and a similar muon kinematics can be found in a recent paper based on $\mathrm{PbPb}$ data collected in 2015 [121], where it is stated that the muon thresholds are $p_{T}>3.5 \mathrm{GeV}$ for $|\eta|<1.2, p_{T}>$ $1.8 \mathrm{GeV}$ for $2.1<|\eta|<2.4$, and linearly interpolated in the intermediate $|\eta|$ region. In order to keep things simple and less specific to the geometry of a specific detector, we use the conservative estimate $3 \mathrm{GeV}$.
}

subdominant change due to the fact that the momentum distributions in heavy-ion collisions are slightly different. Therefore, we adapt the value $f_{\text {cut }}=0.1$ obtained from fitting the simplified detector model (12) to the simulation.

We finally take account of the Lorentz factor in the model (16) by expressing $\beta \gamma$ for each choice of $M$ in terms of the $B$-meson momentum in the laboratory frame and the angle between this momentum and the $N$ momentum. We average Eq. (16) over both, using a flat prior for the angle in the $B$ rest frame, and adopt $B$-meson spectra that we have determined by generating the process $p p \rightarrow \bar{b} b$ with up to one additional jet using MadGraph5_aMC@NLO with subsequent hadronization and matching of soft jets with PYTHIA.

\section{Results}

We present the results of our computation in Fig. 9, where we compare the sensitivity that can be achieved in proton and heavy-ion collisions for equal running time using the luminosities as in Sec. VA. The results show that data from $\mathrm{PbPb}$ collisions could improve existing bounds on the properties of heavy neutrinos by more than an order of magnitude. Furthermore, for ArAr collisions, the combined enhancement due to the larger number of nucleons, point (i), and the lower cut in $p_{T}$, point (iii), can overcompensate for the effect of the lower instantaneous luminosity compared to proton collisions, and one can achieve a better sensitivity per unit of running time. Here we have not taken advantage of the absence of pileup at all, i.e., point (ii), and we recall that we made a very conservative estimate of the effective $\sigma_{B}$. This suggests that, in the range $2 \mathrm{GeV}<M<5 \mathrm{GeV}$ and using the sensitivities estimated by CERN's Physics Beyond Colliders Working Group [9], ArAr collisions with $L=$ $5 \mathrm{pb}^{-1}$ could achieve a higher sensitivity than FASER2 with $L=3 \mathrm{ab}^{-1}$ and a comparable sensitivity as CODEXb [16] with $L=300 \mathrm{fb}^{-1}$, while MATHUSLA [13-15] with $L=3 \mathrm{ab}^{-1}$ would be more than an order of magnitude more sensitive. Also the SHiP experiment $[137,138]$ with $2 \times 10^{20}$ protons on target could achieve a higher sensitivity. However, no decision has been made so far about the construction of these proposed future detectors. For FASER the first phase has been approved, which is almost an order of magnitude less sensitive than FASER2 [139].

\section{DISCUSSION}

We propose to search for LLPs via displaced vertex searches in heavy-ion collisions at the LHC. In the context of LLP searches heavy-ion collisions provide four main advantages in comparison to $p p$ collisions: (i) The number of parton level interactions per hadron-hadron collision is larger, (ii) there is no pileup, which, e.g., renders the probability of misidentifying the primary vertex practically negligible, (iii) the lower instantaneous luminosity makes it 


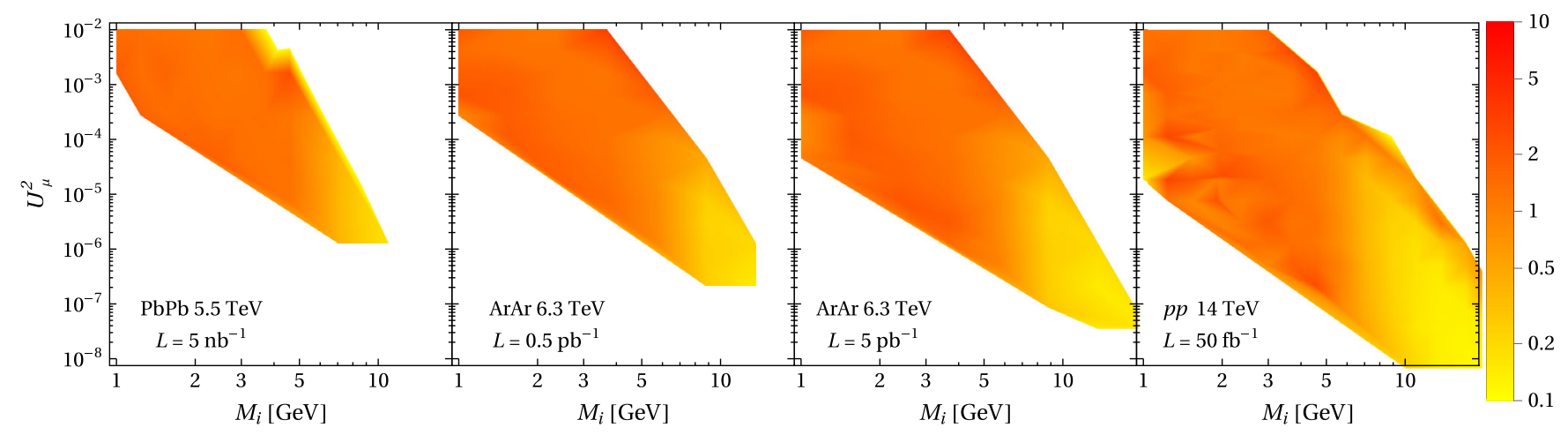

FIG. 8. Ratio between the simplified detector model (12) and the number of events predicted by the simulations performed in Sec. V A, for $\mathrm{PbPb}, \mathrm{ArAr}$ (pessimistic and optimistic), and $p p$ at 5.5, 6.3, and $14 \mathrm{TeV}$, respectively. The displayed region in the plane of heavy neutrino mass $M$ and mixing $U_{\mu}^{2}$ corresponds to parameter values where the model (12) predicts more than 0.1 events.

possible to considerably loosen the triggers used in the main detectors, and (iv) there are new production mechanisms The track multiplicity, which is traditionally considered to be a reason that speaks against new physics searches in heavy-ion collisions, is not considerably higher than in high pileup $p p$ collisions, leaving the lower instantaneous luminosity as the main disadvantage.

\section{A. Summary of the main results}

In the present work, we focus on points (i) and (iii), using the specific case of heavy neutrinos with masses in the $\mathrm{GeV}$ range as an illustrative example. We consider two production mechanisms of heavy neutrinos, production in $W$-boson decay and in $B$-meson decay. If the same cuts are applied as in $p p$ collisions we find that the limitations on the instantaneous luminosity for $\mathrm{PbPb}$ suppress the observable number of events per unit of run-time by almost 2 orders of magnitude. This suppression can be reduced to less than 1 order of magnitude for lighter nuclei, the use of those is currently explored by the heavy-ion community for other reasons [47] such as the longer beam lifetime.

For the production in $W$-boson decays this means that heavy-ion collisions in general do not offer a competitive alternative to searches in proton collisions, though the

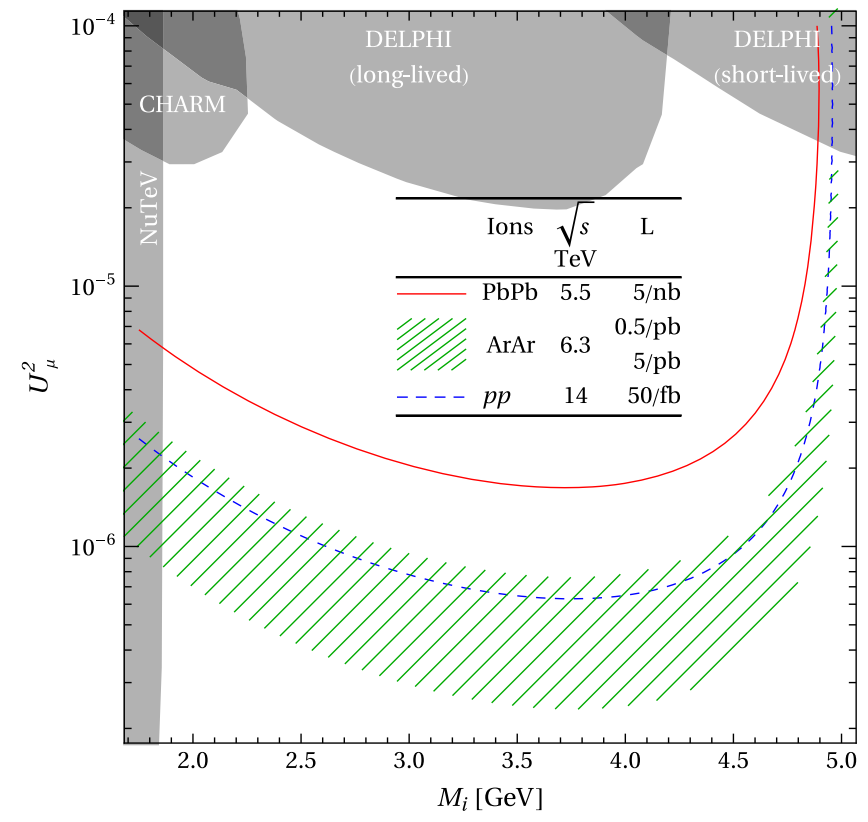

(a) Exclusion with $2 \sigma$.

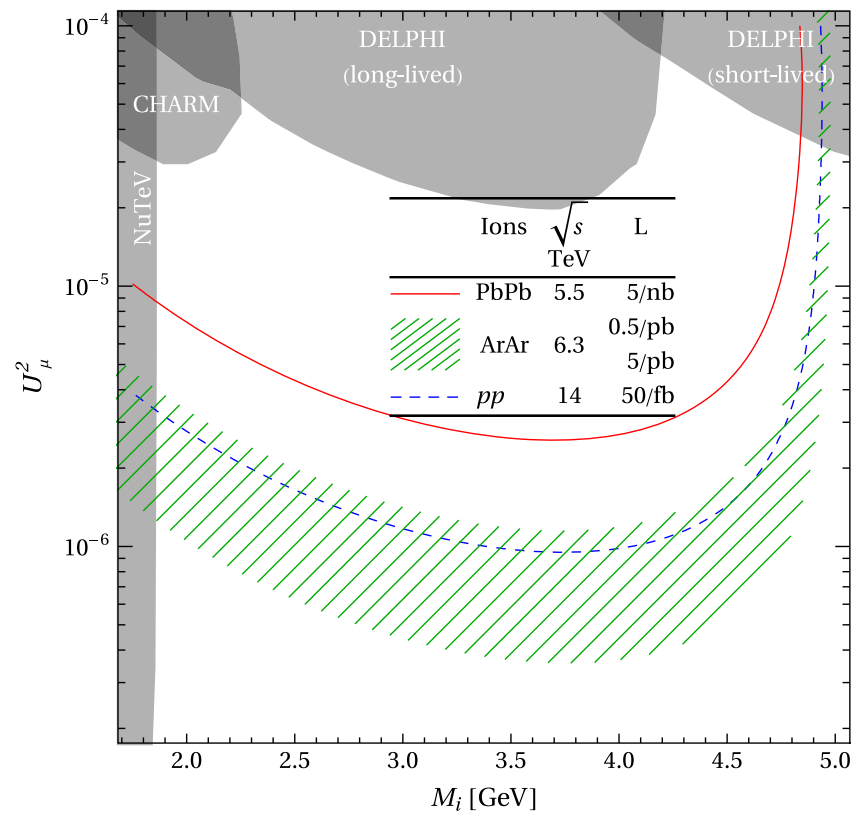

(b) Discovery with $5 \sigma$.

FIG. 9. Sensitivity for heavy neutrinos produced in $B$ decays with the same conventions as in Fig. 5. (a) and (b) correspond to exclusion (four events) and discovery (nine events), respectively. The results were obtained from the simplified detector model (16) as described in Sec. V B. 
integrated luminosity of the HL-LHC in ArAr collisions would be sufficient to push the sensitivity far beyond current experimental limits, see Fig. 5. Loosening the triggers for $N$ produced in $W$ decays only leads to a marginal improvement. The situation is much more promising when considering the production in $B$-meson decays, which leads to a larger number of events, but signatures with much lower $p_{T}$. The results shown in Fig. 9 are remarkable in several ways.

First, data from the complete $\mathrm{PbPb}$ run could improve the sensitivity of searches for heavy neutrinos by more than an order of magnitude in comparison to current bounds. For a small range of masses over $4 \mathrm{GeV}$ the improvement would amount to 2 orders of magnitude. If the LHC's heavy-ion runs were performed with Ar instead, the improvement would be up to 3 orders of magnitude.

Second, the sensitivity that could be achieved in a given unit of running time is actually larger in ArAr collisions than in proton collisions due to the lower cuts on $p_{T}$ that can be imposed. This is not sufficient to entirely compensate for the longer scheduled running time for proton collisions. However, we did not take advantage of the absence of pileup, point (ii), in the present analysis. This suggests that for models where pileup poses a serious problem for the extraction of signatures, see, e.g., Fig. 1, heavy-ion collisions could actually be more sensitive than proton collisions.

Finally, heavy-ion collisions would allow one to study the properties of long-lived particles in a very different environment than proton collisions. This can be particularly interesting for cosmologically motivated LLPs because this environment roughly resembles the primordial plasma that filled the early Universe. The properties of some new particles, such as axionlike particles, are expected to change qualitatively during the transition from QGP to hadronic matter. Others, e.g., sexaquarks, would be primarily produced during this transition in the early Universe, hence the production in heavy-ion collisions would resemble the mechanism that generated them cosmologically [10]. For the specific case of heavy neutrinos in the $\nu \mathrm{MSM}$ considered here, a study of the heavy neutrino properties in the QGP could help to shed light on their potential impact on dark matter production, as discussed in Sec. VIC.

\section{B. Complementary approaches}

Heavy-ion collisions are not the only way to search for low $p_{T}$ events in the LHC main detectors. Another opportunity is offered by the so-called " $B$ parking" data of CMS, pioneered at the end of Run 2 [140,141]. The parking concept consists of storing for later processing (during a long shutdown) a fraction of the data passing mild thresholds. With the data parked in 2018, CMS is expected to add $10^{10}$ events with low $p_{T} B$ mesons [142] that did not pass the standard trigger paths. The same order of magnitude is expected to be achievable by CMS at the end of each run. ${ }^{6}$ This should be compared with our estimation of the $p p$ yearly dataset in HL-LHC, amounting to around $7 \times 10^{10} B$ mesons passing standard triggers. We are not considering in our study any additional contribution from parking, as there are no firm plans for parking in future LHC runs, and future storage capacities are difficult to estimate. We remark, though, that there is no fundamental limitation preventing the same concept to be used also for heavy-ion runs, which in the context of our proposal may mean enlarging the dataset with further trigger paths, allowing one to consider additional signatures (e.g., an electron and a muon, or a muon and a fully reconstructed hadronic final state). We do not elaborate further, as that would crucially depend on the details of future implementations of the parking concept.

Alternatively, as proposed in [143], the recorded pileup events could be exploited to discover light new physics. The number of additional useful events can be written as $N_{\mathrm{PU}}=\langle\mathrm{PU}\rangle w t r$, where $\langle\mathrm{PU}\rangle$ is the average pileup, $w$ is the trigger bandwidth, $t$ is the running time, and $r=\sigma_{B}^{p p} / \sigma_{\text {tot }}^{p p}$ indicates the ratio of cross sections between $B$-meson production and the total inelastic $p p$ cross section. For $p p$ runs at the HL-LHC we assume $\langle\mathrm{PU}\rangle \approx 200$ and use for $w$ the stated goal to record $7.5 \mathrm{kHz}$ on tape [144]. Based on past experience, we assume a running time of $t \approx 10^{7} \mathrm{~s}$ each year. Finally, we calculated $\sigma_{B}^{p p} \approx 1.2 \times 10^{8} \mathrm{pb}$ for $B$ mesons produced with $p_{T}>3 \mathrm{GeV}$ (see Sec. VB 2), which divided by $\sigma_{\mathrm{tot}}^{p p} \approx 80 \mathrm{mb}$ [145] yields $r \approx$ $1.5 \times 10^{-3}$. Hence, pileup results in $N_{\mathrm{PU}} \approx 2.25 \times 10^{10}$ additional $B$ mesons that could be exploited in the future to enhance the useful statistics of the $p p$ data for our purposes. While this additional statistics is not insignificant, it is still affected by all limitations of high-luminosity $p p$ runs that are of relevance for our proposal (but not addressed quantitatively in this study) due to the ambiguity to associate the final state to its original production vertex.

Finally, one may wonder whether asymmetric collisions between protons and heavy ions may offer advantages for LLP searches. Compared to $\mathrm{PbPb}$ collisions, a much larger luminosity and nucleon center-of-mass energy can be achieved in $p \mathrm{~Pb}$ collisions [24], while maintaining the advantage of being free of pileup. However, the cross section is also reduced as the multiplicative factor in partonic cross section only scales as $A$ instead of $A^{2}$. Therefore, we estimate that a search in $p \mathrm{~Pb}$ collisions would not be more sensitive than one in $\mathrm{PbPb}$ collisions. We have checked that the increase in center-of-mass energy has only a marginal effect on the processes we have considered.

\footnotetext{
${ }^{6}$ As the parked data can only be processed during a long shutdown, parked triggers are expected to be executed only during the last year of each run.
} 


\section{Model-dependent remarks}

We have used the example of heavy neutrinos to illustrate the potential of heavy-ion collisions to search for new particles. We chose this model for two reasons. First, its phenomenology is very well known, and some of us have studied similar signatures as the ones considered here in proton collisions [76]. This has the advantage that we are in a good position to isolate the specific effects of heavy-ion collisions from other uncertainties in the study. Second, the choice of the model is conservative with respect to the comparison between proton and heavy-ion collisions because it only takes advantage of two out of the four benefits (i)-(iv).

Since the heavy neutrinos here only act as an illustrative example, we refrain from going into too much detail about the model itself and its phenomenology. We only summarize the most relevant information needed to put the sensitivity lines in Fig. 9 into context and refer the interested reader to Ref. [109] as well as the more recent reviews cited in footnote 1 .

Light neutrino oscillation data and the baryon asymmetry of the Universe can be explained in the entire white part of the plots in Figs. 5 and 9 if there are at least three flavors of heavy neutrinos [146]. ${ }^{7}$ Heavy neutrinos in the mass range considered here can indirectly affect the production of sterile neutrino dark matter by generating chemical potentials that trigger a resonant enhancement of the dark matter production [151], but current studies suggest that the required magnitude of these potentials [152-154] can only be generated for mixing angles that are too small to be accessed by the searches we propose $[107,108,155]$.

In addition to an improved sensitivity in the GeV mass region, heavy-ion collisions can also help to shed light on the role of heavy neutrinos in cosmology because they offer an opportunity to study their properties in a dense plasma that roughly resembles the early Universe. The generation of lepton asymmetries at temperatures below the electroweak scale is highly sensitive to their mass splitting $[107,108,155,156]$, which is subject to thermal corrections. These late time asymmetries would not affect the baryon asymmetry of the Universe, but can lead to the aforementioned resonant production of dark matter [151,152] that manifests itself in observable modifications of the matter

\footnotetext{
${ }^{7}$ For two heavy neutrinos baryogenesis requires roughly $\left(U^{2} / 10^{-5}\right)(M / \mathrm{GeV})<1[97,147,148]$ and is only possible for specific flavor mixing patterns $[97,149,150]$. These restrictions are lifted for three or more right-handed neutrino flavors [146].
}

power spectrum, see $[66,67]$ and references therein. Hence, heavy-ion collisions at least in principle can offer a indirect probe of the dark matter production mechanism in the $\nu \mathrm{MSM}$, though it should be added that it is not clear whether the in-medium properties of the heavy neutrinos could be probed at a sufficient accuracy to draw any definite conclusions in the foreseeable future.

\section{CONCLUSION}

In summary, we find that heavy-ion collisions provide a promising way to explore regions of the parameter space of hidden sector models that are hard to probe in proton collisions. We show this explicitly for heavy neutrino searches in the $\nu \mathrm{MSM}$. For this study we only take advantage of the fact that the LHC main detectors can be operated with looser triggers in heavy-ion collisions than in high intensity proton collisions, which improves the sensitivity to LLPs that decay into particles with low $p_{T}$. Another advantage of heavy-ion collisions is the absence of pileup, which, e.g., entirely avoids the problem of vertex misidentification, i.e., eliminates a systematic limitation in LLP searches with nontrivial event topology. We postpone a more detailed study of this aspect to future work. In addition to this, it is well known that heavy-ion collisions can offer entirely new production mechanisms that are absent in proton collisions. In combination, this provides strong motivation to include potential new physics searches in the discussion of the future of the heavy-ion program at CERN [10].

\section{ACKNOWLEDGMENTS}

We thank Olivier Mattelaer for his valuable contribution to the early stages of the work. We thank Georgios Konstantinos Krintiras, John Jowett, Fabio Maltoni, Emilien Chapon, Jessica Prisciandaro, Mauro Verzetti, Martino Borsato, Elena Graverini, Giacomo Bruno, and Matteo Cacciari for very helpful discussions as well as Albert de Roeck and Steven Lowette for pointing us to relevant material. Some of the figures in this article have been reproduced or adapted from [18]. We appreciated Fosco Loregian's suggestions on how to improve Fig. 1. This work was partly supported by the F.R.S.-FNRS under Excellence of Science (EOS) Project No. 30820817 (be.h). This project has received funding from the European Union's Horizon 2020 research and innovation program under Marie Skłodowska-Curie Grant Agreement No. 750627. 
[1] G. 't Hooft, Naturalness, chiral symmetry, and spontaneous chiral symmetry breaking, NATO Sci. Ser., Ser. B 59, 135 (1980).

[2] S. Weinberg, The problem of mass, Trans. N.Y. Acad. Sci. 38, 185 (1977).

[3] A. A. Belavin, A. M. Polyakov, A. S. Schwartz, and Y. S. Tyupkin, Pseudoparticle solutions of the Yang-Mills equations, Phys. Lett. 59B, 85 (1975).

[4] G. 't Hooft, Symmetry Breaking Through Bell-Jackiw Anomalies, Phys. Rev. Lett. 37, 8 (1976).

[5] R. Jackiw and C. Rebbi, Vacuum Periodicity in a YangMills Quantum Theory, Phys. Rev. Lett. 37, 172 (1976).

[6] Y. B. Zeldovich, Cosmological constant and elementary particles, Pisma Zh. Eksp. Teor. Fiz. 6, 883 (1967) [JETP Lett. 6, 316 (1967)].

[7] S. Weinberg, The cosmological constant problem, Rev. Mod. Phys. 61, 1 (1989).

[8] G. F. Giudice, Naturally speaking: The naturalness criterion and physics at the LHC, in Perspectives on LHC Physics, edited by G. Kane and A. Pierce (World Scientific, Singapore, 2008), pp. 155-178.

[9] J. Beacham et al., Physics beyond colliders at CERN: Beyond the Standard Model working group report, J. Phys. G 47, 010501 (2020).

[10] R. Bruce et al., New physics searches with heavy-ion collisions at the LHC, arXiv:1812.07688.

[11] J. Alimena et al., Searching for long-lived particles beyond the Standard Model at the Large Hadron Collider, arXiv:1903.04497.

[12] J. L. Feng, I. Galon, F. Kling, and S. Trojanowski, ForwArd Search ExpeRiment at the LHC, Phys. Rev. D 97, 035001 (2018).

[13] J. P. Chou, D. Curtin, and H. J. Lubatti, New detectors to explore the lifetime frontier, Phys. Lett. B 767, 29 (2017).

[14] D. Curtin et al., Long-lived particles at the energy frontier: The MATHUSLA physics case, Rep. Prog. Phys. 82, 116201 (2019).

[15] C. Alpigiani et al., A letter of intent for MATHUSLA: A dedicated displaced vertex detector above ATLAS or CMS, CERN Report No. CERN-LHCC-2018-025, 2018.

[16] V. V. Gligorov, S. Knapen, M. Papucci, and D. J. Robinson, Searching for long-lived particles: A compact detector for exotics at LHCb, Phys. Rev. D 97, 015023 (2018).

[17] V. V. Gligorov, S. Knapen, B. Nachman, M. Papucci, and D. J. Robinson, Leveraging the ALICE/L3 cavern for long-lived particle searches, Phys. Rev. D 99, 015023 (2019).

[18] M. Drewes, A. Giammanco, J. Hajer, M. Lucente, and O. Mattelaer, Searching for New Long-Lived Particles in Heavy-Ion Collisions at the LHC, Phys. Rev. Lett. 124, 081801 (2020).

[19] N. Cartiglia, Measurement of the proton-proton total, elastic, inelastic and diffractive cross sections at 2, 7, 8 and $57 \mathrm{TeV}$, arXiv:1305.6131.

[20] A. M. Sirunyan et al. (CMS Collaboration), Measurement of the inclusive $t \bar{t}$ cross section in $p p$ collisions at $\sqrt{s}=$ 5.02 TeV using final states with at least one charged lepton, J. High Energy Phys. 03 (2018) 115.
[21] M. Cacciari, M. Greco, and P. Nason, The $p_{T}$ spectrum in heavy flavor hadroproduction, J. High Energy Phys. 05 (1998) 007.

[22] M. Cacciari, S. Frixione, and P. Nason, The $p_{T}$ spectrum in heavy flavor photoproduction, J. High Energy Phys. 03 (2001) 006.

[23] M. Cacciari, M. L. Mangano, and P. Nason, Gluon PDF constraints from the ratio of forward heavy-quark production at the LHC at $\sqrt{s}=7$ and $13 \mathrm{TeV}$, Eur. Phys. J. C 75, 610 (2015).

[24] Z. Citron et al., Future physics opportunities for highdensity QCD at the LHC with heavy-ion and proton beams, CERN Yellow Rep.: Monogr. 7 (2019).

[25] A. J. Baltz, The physics of ultraperipheral collisions at the LHC, Phys. Rep. 458, 1 (2008).

[26] O. Gould and A. Rajantie, Magnetic Monopole Mass Bounds from Heavy-Ion Collisions and Neutron Stars, Phys. Rev. Lett. 119, 241601 (2017).

[27] S. Knapen, T. Lin, H. K. Lou, and T. Melia, Searching for Axionlike Particles with Ultraperipheral Heavy-Ion Collisions, Phys. Rev. Lett. 118, 171801 (2017).

[28] J. Alwall, R. Frederix, S. Frixione, V. Hirschi, F. Maltoni, O. Mattelaer, H. S. Shao, T. Stelzer, P. Torrielli, and M. Zaro, The automated computation of tree-level and nextto-leading order differential cross sections, and their matching to parton shower simulations, J. High Energy Phys. 07 (2014) 079.

[29] A. M. Sirunyan et al. (CMS Collaboration), Pseudorapidity distributions of charged hadrons in xenon-xenon collisions at $\sqrt{s_{\mathrm{NN}}}=5.44 \mathrm{TeV}$, Phys. Lett. B 799, 135049 (2019).

[30] J. Adam et al. (ALICE Collaboration), Centrality Dependence of the Charged-Particle Multiplicity Density at Midrapidity in $\mathrm{Pb}-\mathrm{Pb}$ Collisions at $\sqrt{s_{\mathrm{NN}}}=5.02 \mathrm{TeV}$, Phys. Rev. Lett. 116, 222302 (2016).

[31] V. Khachatryan et al. (CMS Collaboration), Pseudorapidity distribution of charged hadrons in proton-proton collisions at $\sqrt{s}=13 \mathrm{TeV}$, Phys. Lett. B 751, 143 (2015).

[32] M. Aaboud et al. (ATLAS Collaboration), Chargedparticle distributions at low transverse momentum in $\sqrt{\mathrm{s}}=$ $13 \mathrm{TeV} p p$ interactions measured with the ATLAS detector at the LHC, Eur. Phys. J. C 76, 502 (2016).

[33] J. Adam et al. (ALICE Collaboration), Pseudorapidity and transverse-momentum distributions of charged particles in proton-proton collisions at $\sqrt{s}=13 \mathrm{TeV}$, Phys. Lett. B 753, 319 (2016).

[34] G. Apollinari, I. B. Alonso, O. Brüning, M. Lamont, and L. Rossi, High-Luminosity Large Hadron Collider (HL-LHC): Preliminary design report, 285 (2015).

[35] G. Apollinari, O. Brüning, T. Nakamoto, and L. Rossi, High Luminosity Large Hadron Collider HL-LHC, CERN Yellow Report No. CERN-2015-005, 2015.

[36] ATLAS Collaboration, ATLAS phase-II upgrade scoping document, Reports No. CERN-LHCC-2015-020 and No. LHCC-G-166, 2015.

[37] D. Contardo et al. (CMS Collaboration), Technical proposal for the phase-II upgrade of the CMS detector, Reports No. CERN-LHCC-2015-010, No. LHCC-P-008, and No. CMS-TDR-15-02, 2015. 
[38] A. M. Sirunyan et al. (CMS Collaboration), Observation of Top Quark Production in Proton-Nucleus Collisions, Phys. Rev. Lett. 119, 242001 (2017).

[39] CMS Collaboration, Evidence for top quark production in nucleus-nucleus collisions, Report No. CMS-PAS-HIN19-001, 2019.

[40] M. Schaumann, Heavy-ion performance of the LHC and future colliders, Ph.D. Thesis, RWTH Aachen University, 2015.

[41] H. H. Braun, A. Fassò, A. Ferrari, J. M. Jowett, P. R. Sala, and G. I. Smirnov, Hadronic and electromagnetic fragmentation of ultrarelativistic heavy ions at LHC, Phys. Rev. ST Accel. Beams 17, 021006 (2014).

[42] J. M. Jowett, M. Schaumann, and R. Versteegen, Heavyion operation of HL-LHC, Adv. Ser. Dir. High Energy Phys. 24, 359 (2015).

[43] J. M. Jowett, M. Schaumann, and R. Versteegen, Heavy ion operation from Run 2 to HL-LHC, in Proceedings of RLIUP: Review of LHC and Injector Upgrade Plans, Archamps, France, 2013, edited by B. Goddard and F. Zimmermann (CERN, Geneva, 2014), https://doi.org/ 10.5170/CERN-2014-006.167.

[44] J. Jowett, Colliding heavy ions in the LHC, in Proceedings of the 9th International Particle Accelerator Conference (IPAC 2018), Vancouver, British Columbia, Canada, edited by S. Koscielniak (JACoW, Geneva, 2018), https://doi.org/10.18429/JACoW-IPAC2018-TUXGBD2.

[45] K. Aamodt et al. (ALICE Collaboration), The ALICE experiment at the CERN LHC, J. Instrum. 3, S08002 (2008).

[46] Y. Zhang (LHCb Collaboration), Results on heavy ion collisions at LHCb, LHCb Report No. LHCb-PROC-2016013, 2016.

[47] J. Jowett, HL-LHC performance: Update for HE-LHC and light ions, in Proceedings of the Workshop on the Physics of HL-LHC, and Perspectives at HE-LHC (CERN, Geneva, 2018), https://indico.cern.ch/event/686494/contributions/ $3034628 /$.

[48] M. Benedikt, D. Schulte, and F. Zimmermann, Optimizing integrated luminosity of future hadron colliders, Phys. Rev. ST Accel. Beams 18, 101002 (2015).

[49] J. C. Pati and A. Salam, Lepton number as the fourth color, Phys. Rev. D 10, 275 (1974).

[50] R. N. Mohapatra and J. C. Pati, A natural left-right symmetry, Phys. Rev. D 11, 2558 (1975).

[51] G. Senjanovic and R. N. Mohapatra, Exact left-right symmetry and spontaneous violation of parity, Phys. Rev. D 12, 1502 (1975).

[52] M. Nemevšek, F. Nesti, and J. C. Vasquez, Majorana Higgses at colliders, J. High Energy Phys. 04 (2017) 114.

[53] M. Drewes, The phenomenology of right handed neutrinos, Int. J. Mod. Phys. E 22, 1330019 (2013).

[54] P. Minkowski, $\mu \rightarrow e \gamma$ at a rate of one out of $10^{9}$ muon decays?, Phys. Lett. 67B, 421 (1977).

[55] M. Gell-Mann, P. Ramond, and R. Slansky, Complex spinors and unified theories, Conf. Proc. C 790927, 315 (1979).

[56] R. N. Mohapatra and G. Senjanovic, Neutrino Mass and Spontaneous Parity Violation, Phys. Rev. Lett. 44, 912 (1980).
[57] T. Yanagida, Horizontal symmetry and masses of neutrinos, Prog. Theor. Phys. 64, 1103 (1980).

[58] J. Schechter and J. W.F. Valle, Neutrino masses in $\mathrm{SU}(2) \times \mathrm{U}(1)$ theories, Phys. Rev. D 22, 2227 (1980).

[59] J. Schechter and J. W. F. Valle, Neutrino decay and spontaneous violation of lepton number, Phys. Rev. D 25, 774 (1982).

[60] M. Fukugita and T. Yanagida, Baryogenesis without grand unification, Phys. Lett. B 174, 45 (1986).

[61] S. Dodelson and L. M. Widrow, Sterile Neutrinos as Dark Matter, Phys. Rev. Lett. 72, 17 (1994).

[62] K. N. Abazajian et al., Light sterile neutrinos: A white paper, arXiv:1204.5379.

[63] I. Brivio and M. Trott, Radiatively Generating the Higgs Potential and Electroweak Scale via the Seesaw Mechanism, Phys. Rev. Lett. 119, 141801 (2017).

[64] L. Canetti, M. Drewes, and M. Shaposhnikov, Matter and antimatter in the Universe, New J. Phys. 14, 095012 (2012).

[65] E. J. Chun et al., Probing leptogenesis, Int. J. Mod. Phys. A 33, 1842005 (2018).

[66] M. Drewes et al., A white paper on keV sterile neutrino dark matter, J. Cosmol. Astropart. Phys. 01 (2017) 025.

[67] A. Boyarsky, M. Drewes, T. Lasserre, S. Mertens, and O. Ruchayskiy, Sterile neutrino dark matter, Prog. Part. Nucl. Phys. 104, 1 (2019).

[68] A. Atre, T. Han, S. Pascoli, and B. Zhang, The search for heavy majorana neutrinos, J. High Energy Phys. 05 (2009) 030.

[69] F. F. Deppisch, P. S. B. Dev, and A. Pilaftsis, Neutrinos and collider physics, New J. Phys. 17, 075019 (2015).

[70] Y. Cai, T. Han, T. Li, and R. Ruiz, Lepton number violation: Seesaw models and their collider tests, Front. Phys. 6, 40 (2018).

[71] S. Antusch, E. Cazzato, and O. Fischer, Sterile neutrino searches at future $e^{-} e^{+}, p p$, and $e^{-} p$ colliders, Int. J. Mod. Phys. A 32, 1750078 (2017).

[72] J. Gluza, On teraelectronvolt Majorana neutrinos, Acta Phys. Pol. B 33, 1735 (2002).

[73] M. Shaposhnikov, A possible symmetry of the $2 \mathrm{MSM}$, Nucl. Phys. B763, 49 (2007).

[74] J. Kersten and A. Y. Smirnov, Right-handed neutrinos at CERN LHC and the mechanism of neutrino mass generation, Phys. Rev. D 76, 073005 (2007).

[75] K. Moffat, S. Pascoli, and C. Weiland, Equivalence between massless neutrinos and lepton number conservation in fermionic singlet extensions of the Standard Model, arXiv:1712.07611.

[76] M. Drewes and J. Hajer, Heavy neutrinos in displaced vertex searches at the LHC and HL-LHC, arXiv:1903. 06100.

[77] C. Dib and C.S. Kim, Remarks on the lifetime of sterile neutrinos and the effect on detection of rare meson decays $M^{+} \rightarrow M^{\prime}-\ell^{+} \ell^{+}$, Phys. Rev. D 89, 077301 (2014).

[78] J. C. Helo, M. Hirsch, and S. Kovalenko, Heavy neutrino searches at the LHC with displaced vertices, Phys. Rev. D 89, 073005 (2014).

[79] E. Izaguirre and B. Shuve, Multilepton and lepton jet probes of sub-weak-scale right-handed neutrinos, Phys. Rev. D 91, 093010 (2015). 
[80] A. M. Gago, P. Hernández, J. Jones-Pérez, M. Losada, and A. M. Briceño, Probing the type I seesaw mechanism with displaced vertices at the LHC, Eur. Phys. J. C 75, 470 (2015).

[81] C. O. Dib and C.S. Kim, Discovering sterile neutrinos lighter than $M_{W}$ at the LHC, Phys. Rev. D 92, 093009 (2015).

[82] G. Cvetic and C.S. Kim, Rare decays of $B$ mesons via onshell sterile neutrinos, Phys. Rev. D 94, 053001 (2016).

[83] G. Cottin, J. C. Helo, and M. Hirsch, Searches for light sterile neutrinos with multitrack displaced vertices, Phys. Rev. D 97, 055025 (2018).

[84] S. Antusch, E. Cazzato, and O. Fischer, Sterile neutrino searches via displaced vertices at LHCb, Phys. Lett. B 774, 114 (2017).

[85] G. Cottin, J. C. Helo, and M. Hirsch, Displaced vertices as probes of sterile neutrino mixing at the LHC, Phys. Rev. D 98, 035012 (2018).

[86] A. Abada, N. Bernal, M. Losada, and X. Marcano, Inclusive displaced vertex searches for heavy neutral leptons at the LHC, J. High Energy Phys. 01 (2019) 093.

[87] K. Bondarenko, A. Boyarsky, M. Ovchynnikov, O. Ruchayskiy, and L. Shchutska, Probing new physics with displaced vertices: Muon tracker at CMS, Phys. Rev. D 100, 075015 (2019).

[88] J. Liu, Z. Liu, L.-T. Wang, and X.-P. Wang, Seeking for sterile neutrinos with displaced leptons at the LHC, J. High Energy Phys. 07 (2019) 159.

[89] C. O. Dib, C. S. Kim, and S. T. Araya, Search for light sterile neutrinos from $W^{ \pm}$decays at the LHC, arXiv: 1903 . 04905.

[90] C. O. Dib, C. S. Kim, N. A. Neill, and X.-B. Yuan, Search for sterile neutrinos decaying into pions at the LHC, Phys. Rev. D 97, 035022 (2018).

[91] G. Cvetič, A. Das, and J. Zamora-Saá, Probing heavy neutrino oscillations in rare $W$ boson decays, J. Phys. G 46, 075002 (2019).

[92] G. Cvetič, A. Das, S. Tapia, and J. Zamora-Saá, Measuring the heavy neutrino oscillations in rare $W$ boson decays at the Large Hadron Collider, J. Phys. G 47, 015001 (2020).

[93] G. Cvetič and C.S. Kim, Sensitivity bounds on heavy neutrino mixing $\left|U_{\mu N}\right|^{2}$ and $\left|U_{\tau N}\right|^{2}$ from LHCb upgrade, Phys. Rev. D 100, 015014 (2019).

[94] A. Blondel, E. Graverini, N. Serra, and M. Shaposhnikov, Search for heavy right handed neutrinos at the FCC-ee, Nucl. Part. Phys. Proc. 273-275, 1883 (2016).

[95] S. Antusch and O. Fischer, Testing sterile neutrino extensions of the Standard Model at future lepton colliders, J. High Energy Phys. 05 (2015) 053.

[96] S. Antusch, E. Cazzato, and O. Fischer, Displaced vertex searches for sterile neutrinos at future lepton colliders, J. High Energy Phys. 12 (2016) 007.

[97] S. Antusch, E. Cazzato, M. Drewes, O. Fischer, B. Garbrecht, D. Gueter, and J. Klaric, Probing leptogenesis at future colliders, J. High Energy Phys. 09 (2018) 124.

[98] M. L. Graesser, Experimental constraints on Higgs boson decays to TeV-scale right-handed neutrinos, arXiv:0705. 2190 .
[99] M. L. Graesser, Broadening the Higgs boson with righthanded neutrinos and a higher dimension operator at the electroweak scale, Phys. Rev. D 76, 075006 (2007).

[100] A. Maiezza, M. Nemevšek, and F. Nesti, Lepton Number Violation in Higgs Decay at LHC, Phys. Rev. Lett. 115, 081802 (2015).

[101] B. Batell, M. Pospelov, and B. Shuve, Shedding light on neutrino masses with dark forces, J. High Energy Phys. 08 (2016) 052.

[102] A. Caputo, P. Hernandez, J. Lopez-Pavon, and J. Salvado, The seesaw portal in testable models of neutrino masses, J. High Energy Phys. 06 (2017) 112.

[103] M. Nemevšek, F. Nesti, and G. Popara, Keung-Senjanović process at the LHC: From lepton number violation to displaced vertices to invisible decays, Phys. Rev. D 97, 115018 (2018).

[104] G. Cottin, J. C. Helo, M. Hirsch, and D. Silva, Revisiting the LHC reach in the displaced region of the minimal left-right symmetric model, Phys. Rev. D 99, 115013 (2019).

[105] T. Asaka, S. Blanchet, and M. Shaposhnikov, The $\nu$ MSM, dark matter and neutrino masses, Phys. Lett. B 631, 151 (2005).

[106] T. Asaka and M. Shaposhnikov, The $\nu$ MSM, dark matter and baryon asymmetry of the Universe, Phys. Lett. B 620 , 17 (2005).

[107] L. Canetti, M. Drewes, and M. Shaposhnikov, Sterile Neutrinos as the Origin of Dark and Baryonic Matter, Phys. Rev. Lett. 110, 061801 (2013).

[108] L. Canetti, M. Drewes, T. Frossard, and M. Shaposhnikov, Dark matter, baryogenesis and neutrino oscillations from right-handed neutrinos, Phys. Rev. D 87, 093006 (2013).

[109] A. Boyarsky, O. Ruchayskiy, and M. Shaposhnikov, The role of sterile neutrinos in cosmology and astrophysics, Annu. Rev. Nucl. Part. Sci. 59, 191 (2009).

[110] D. Gorbunov and M. Shaposhnikov, How to find neutral leptons of the $\nu$ MSM?, J. High Energy Phys. 10 (2007) 015; Erratum, J. High Energy Phys. 11 (2013) 101.

[111] A. Abada, V. De Romeri, M. Lucente, A. M. Teixeira, and T. Toma, Effective Majorana mass matrix from tau and pseudoscalar meson lepton number violating decays, J. High Energy Phys. 02 (2018) 169.

[112] K. Bondarenko, A. Boyarsky, D. Gorbunov, and O. Ruchayskiy, Phenomenology of GeV-scale heavy neutral leptons, J. High Energy Phys. 11 (2018) 032.

[113] S. Pascoli, R. Ruiz, and C. Weiland, Heavy neutrinos with dynamic jet vetoes: Multilepton searches at $\sqrt{s}=14,27$, and $100 \mathrm{TeV}$, J. High Energy Phys. 06 (2019) 049.

[114] A. Vaitaitis et al. (NuTeV and E815 Collaborations), Search for Neutral Heavy Leptons in a High-Energy Neutrino Beam, Phys. Rev. Lett. 83, 4943 (1999).

[115] F. Bergsma et al. (CHARM Collaboration), A search for decays of heavy neutrinos in the mass range $0.5 \mathrm{GeV}$ to $2.8 \mathrm{GeV}$, Phys. Lett. 166B, 473 (1986).

[116] P. Abreu et al. (DELPHI Collaboration), Search for neutral heavy leptons produced in $Z$ decays, Z. Phys. C 74, 57 (1997).

[117] A. M. Sirunyan et al. (CMS Collaboration), Search for Heavy Neutral Leptons in Events with Three Charged 
Leptons in Proton-Proton Collisions at $\sqrt{s}=13 \mathrm{TeV}$, Phys. Rev. Lett. 120, 221801 (2018).

[118] M. Drewes, On the minimal mixing of heavy neutrinos, arXiv:1904.11959.

[119] M. Cacciari, S. Frixione, N. Houdeau, M. L. Mangano, P. Nason, and G. Ridolfi, Theoretical predictions for charm and bottom production at the LHC, J. High Energy Phys. 10 (2012) 137.

[120] P. M. Nadolsky, H.-L. Lai, Q.-H. Cao, J. Huston, J. Pumplin, D. Stump, W.-K. Tung, and C. P. Yuan, Implications of CTEQ global analysis for collider observables, Phys. Rev. D 78, 013004 (2008).

[121] A. M. Sirunyan et al. (CMS Collaboration), Measurement of the $B^{ \pm}$Meson Nuclear Modification Factor in $\mathrm{Pb}-\mathrm{Pb}$ Collisions at $\sqrt{s_{\mathrm{NN}}}=5.02 \mathrm{TeV}$, Phys. Rev. Lett. 119, 152301 (2017).

[122] J. Ghiglieri and M. Laine, Neutrino dynamics below the electroweak crossover, J. Cosmol. Astropart. Phys. 07 (2016) 015.

[123] M. Chrzaszcz, M. Drewes, T. Gonzalo, J. Harz, S. Krishnamurthy, and C. Weniger, A frequentist analysis of three right-handed neutrinos with GAMBIT, arXiv:1908. 02302.

[124] A. Alloul, N. D. Christensen, C. Degrande, C. Duhr, and B. Fuks, FeynRules 2.0-A complete toolbox for tree-level phenomenology, Comput. Phys. Commun. 185, 2250 (2014).

[125] C. Degrande, O. Mattelaer, R. Ruiz, and J. Turner, Fully automated precision predictions for heavy neutrino production mechanisms at hadron colliders, Phys. Rev. D 94, 053002 (2016).

[126] D. Alva, T. Han, and R. Ruiz, Heavy Majorana neutrinos from $W \gamma$ fusion at hadron colliders, J. High Energy Phys. 02 (2015) 072.

[127] K. J. Eskola, P. Paakkinen, H. Paukkunen, and C. A. Salgado, EPPS16: Nuclear parton distributions with LHC data, Eur. Phys. J. C 77, 163 (2017).

[128] J. Alwall, C. Duhr, B. Fuks, O. Mattelaer, D. G. Öztürk, and C.-H. Shen, Computing decay rates for new physics theories with FeynRules and MadGraph5_aMC@NLO, Comput. Phys. Commun. 197, 312 (2015).

[129] S. Frixione, E. Laenen, P. Motylinski, and B. R. Webber, Angular correlations of lepton pairs from vector boson and top quark decays in Monte Carlo simulations, J. High Energy Phys. 04 (2007) 081.

[130] P. Artoisenet, R. Frederix, O. Mattelaer, and R. Rietkerk, Automatic spin-entangled decays of heavy resonances in Monte Carlo simulations, J. High Energy Phys. 03 (2013) 015.

[131] T. Sjöstrand, S. Ask, J. R. Christiansen, R. Corke, N. Desai, P. Ilten, S. Mrenna, S. Prestel, C. O. Rasmussen, and P.Z. Skands, An introduction to PYTHIA 8.2, Comput. Phys. Commun. 191, 159 (2015).

[132] J. de Favereau, C. Delaere, P. Demin, A. Giammanco, V. Lemaître, A. Mertens, and M. Selvaggi (DELPHES 3 Collaboration), DELPHES 3, a modular framework for fast simulation of a generic collider experiment, J. High Energy Phys. 02 (2014) 057.

[133] B. Smits, Efficiency issues for ray tracing, J. Graph. Tools 3, 1 (1998).
[134] A. Williams, S. Barrus, K. Morley, and P. Shirley, An efficient and robust ray-box intersection algorithm, J. Graph. Tools 10, 49 (2005).

[135] M. Aaboud et al. (ATLAS Collaboration), Search for longlived, massive particles in events with displaced vertices and missing transverse momentum in $\sqrt{s}=13 \mathrm{TeV} p p$ collisions with the ATLAS detector, Phys. Rev. D 97, 052012 (2018).

[136] C. Liu and N. Neumeister, Reconstruction of cosmic and beam-halo muons, Eur. Phys. J. C 56, 449 (2008).

[137] M. Anelli et al. (SHiP Collaboration), A facility to Search for Hidden Particles (SHiP) at the CERN SPS, arXiv: 1504.04956.

[138] S. Alekhin et al., A facility to Search for Hidden Particles at the CERN SPS: The SHiP physics case, Rep. Prog. Phys. 79, 124201 (2016).

[139] A. Ariga et al. (FASER Collaboration), FASER's physics reach for long-lived particles, Phys. Rev. D 99, 095011 (2019).

[140] CMS Collaboration, Data parking and data scouting at the CMS experiment, Report No. CMS-DP-2012-022, 2012.

[141] CMS Collaboration, Recording and reconstructing 10 billion unbiased $b$ hadron decays in CMS, Report No. CMSDP-2019-043, 2019.

[142] J. Duarte, CMS and LHC report, in Proceedings of the All Experimenters' Meeting, 2018, https://indico.fnal.gov/ event/17519/contribution/6/material/slides/0.pdf.

[143] B. Nachman and F. Rubbo, Search strategy using LHC pileup interactions as a zero bias sample, Phys. Rev. D 97, 092002 (2018).

[144] J.-M. André et al., The CMS data acquisition system for the phase-2 upgrade, arXiv:1806.08975.

[145] G. Antchev et al. (TOTEM Collaboration), First measurement of elastic, inelastic and total cross-section at $\sqrt{s}=13 \mathrm{TeV}$ by TOTEM and overview of crosssection data at LHC energies, Eur. Phys. J. C 79, 103 (2019).

[146] A. Abada, G. Arcadi, V. Domcke, M. Drewes, J. Klaric, and M. Lucente, Low-scale leptogenesis with three heavy neutrinos, J. High Energy Phys. 01 (2019) 164.

[147] S. Eijima, M. Shaposhnikov, and I. Timiryasov, Parameter space of baryogenesis in the $\nu$ MSM, J. High Energy Phys. 07 (2019) 077.

[148] I. Boiarska, K. Bondarenko, A. Boyarsky, S. Eijima, M. Ovchynnikov, O. Ruchayskiy, and I. Timiryasov, Probing baryon asymmetry of the Universe at LHC and SHiP, arXiv:1902.04535.

[149] P. Hernández, M. Kekic, J. López-Pavón, J. Racker, and J. Salvado, Testable baryogenesis in seesaw models, J. High Energy Phys. 08 (2016) 157.

[150] M. Drewes, B. Garbrecht, D. Gueter, and J. Klaric, Testing the low scale seesaw and leptogenesis, J. High Energy Phys. 08 (2017) 018.

[151] X.-D. Shi and G. M. Fuller, A New Dark Matter Candidate: Nonthermal Sterile Neutrinos, Phys. Rev. Lett. 82, 2832 (1999).

[152] T. Asaka, M. Laine, and M. Shaposhnikov, Lightest sterile neutrino abundance within the $\nu$ MSM, J. High Energy 
Phys. 01 (2007) 091; Erratum, J. High Energy Phys. 02 (2015) 28(E).

[153] J. Ghiglieri and M. Laine, Improved determination of sterile neutrino dark matter spectrum, J. High Energy Phys. 11 (2015) 171.

[154] T. Venumadhav, F.-Y. Cyr-Racine, K. N. Abazajian, and C. M. Hirata, Sterile neutrino dark matter: Weak interactions in the strong coupling epoch, Phys. Rev. D 94, 043515 (2016).

[155] J. Ghiglieri and M. Laine, Sterile neutrino dark matter via GeV-scale leptogenesis?, J. High Energy Phys. 07 (2019) 078.

[156] M. Shaposhnikov, The $\nu$ MSM, leptonic asymmetries, and properties of singlet fermions, J. High Energy Phys. 08 (2008) 008. 